\title{
belarus-
}

analysen

\section{FRAUEN IN STAAT UND POLITIK IN BELARUS}

\section{ANALYSE}

Frauen in Staat und Politik in Belarus

Veranika Laputska (Polnische Akademie der Wissenschaften in Warschau)

- STATISTIK

Global Gender Gap Report 2017 (Bericht des „World Economic Forum»)

Human Development Report 2016 (Bericht von UNDP) 11

Frauen und Männer in der Republik Belarus, 2016

(Bericht des Nationalen Komitees für Statistik, Belstat) 13

Frauen in den Wahlkommissionen, gewählten Staatsorganen und Ministerien

DOKUMENTATION

Bericht unabhängiger Wahlbeobachter zu den Kommunalwahlen in Belarus

\section{CHRONIK}

6. Dezember 2017 - 5. März 2018

Leibniz-Institut für Ost- und Südosteuropaforschung
Zentrum für Osteuropa- und internationale Studien (ZOiS) $\mathrm{gGmbH}$

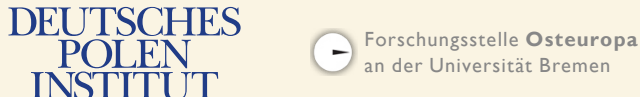

INSTITUT

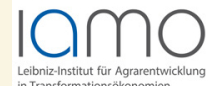




\section{Frauen in Staat und Politik in Belarus}

Veranika Laputska (Polnische Akademie der Wissenschaften in Warschau)

\section{Zusammenfassung}

Am 18. Februar fanden in Belarus Kommunalwahlen statt. Frauen sind traditionell sehr stark in den Wahlkreis- und den territorialen Wahlkommissionen engagiert gewesen, haben an Wahlkampfveranstaltungen teilgenommen und für Abgeordnetenmandate kandidiert, und sogar für das Amt des Präsidenten, wie Tazjana Karatkewitsch im Jahr 2015. Gleichwohl wird die politische Bühne in Belarus größtenteils von Männern dominiert. Obwohl Frauen auf den ersten Blick aktiv an den politischen Prozessen beteiligt sind, bleibt ihre Rolle weit hinter jener von Frauen in etablierten Demokratien zurück.

Der folgende Beitrag behandelt die Rolle der Frauen im aktuellen politischen Geschehen in Belarus. Nicht betrachtet wird der Bereich der regierungstreuen und unabhängigen politischen Parteien sowie der NGO-Sektor, die hinsichtlich der Rolle von Frauen eine andere Dimension aufweisen. Analysiert werden sollen die Entwicklung der Machtverhältnisse zwischen Männern und Frauen in der belarussischen Politik heute sowie deren Vor- und Nachteile.

\section{Frauen bei belarussischen Wahlen}

Seit der Erringung seiner Unabhängigkeit hat Belarus im Bereich der Wahlverwaltung eine Reihe von Transformationen erlebt. In dieser Hinsicht ist die Figur Lidsija Jarmoschyna interessant, die seit 1996 Vorsitzende der belarussischen Zentralen Wahlkommission ist und nach wie vor einer der altgedientesten staatlichen Funktionäre in Belarus.

In der Tat ist sie zu einer der bekanntesten und markantesten Politikerinnen in Belarus geworden, nicht zuletzt wegen ihrer zahlreichen antifeministischen Äußerungen. Das Statement, das ihr eine besonders intensive Aufmerksamkeit der Medien eintrug, war an jene belarussischen Frauen gerichtet, die vor dem Parlamentsgebäude, in dem auch die Zentrale Wahlkommission ihren Sitz hat, gegen den Ausgang der Präsidentschaftswahlen 2010 protestierten. Als die Protestierenden brutal auseinandergejagt wurden und auf einer Pressekonferenz darauf verwiesen wurde, dass unter den Festgenommenen viele Frauen waren, antwortete Lidsija Jarmoschyna, dass »diese `Frauen $`$ zu Hause bleiben sollten und stattdessen Borschtsch [eine traditionelle ostslawische Suppe aus roter Beete] kochen sollten [...] Es ist für Frauen eine Schande, sich an solchen Vorkommnissen zu beteiligen!«. Für diese Äußerung erhielt Frau Jarmoschyna den Preis "Sexist des Jahres 2010« einer feministischen Gruppe.

Ungeachtet solcher Äußerungen der Vorsitzenden der belarussischen Zentralen Wahlkommission beruht die Wahlmaschine in Belarus vor allem auf der Arbeit von Frauen. Belarussische Journalisten haben berechnet, dass während der Präsidentschaftswahlen 2010 die Wahlkommissionen der verschiedenen Ebenen aus 48.307 Frauen und 22.508 Männern (68,2 bzw. 31,8 \%) bestanden. Wadsim Bylina schreibt in einem Artikel über die Zusammensetzung der Wahlkommissionen, dass diese Zahl seit 1999, als der Anteil der Frauen zwischen 60 und 70 Prozent betrug, gestiegen sei .

Als belarussische Medien versuchten, den Gründen für dieses Zahlenverhältnis nachzugehen, stellten sie fest, dass die Wahlkommissionen meist in Schulen oder anderen staatlichen Einrichtungen untergebracht waren, weswegen die Mitglieder der Wahlkommissionen vor allem Lehrer, also Lehrerinnen waren. Im September 2010 hatte es einen Versuch gegeben, die Möglichkeit auszuschließen, dass Lehrer Mitglied einer Wahlkommission werden, da sie von ihren Vorgesetzten weitgehend abhängig sind und »keine Rechte haben«. Der Versuch schlug jedoch fehl. Die Praxis, Lehrer in Wahlkommissionen zu berufen, hielt auch in späteren Jahren an.

Der Beruf des Lehrers (also vor allem der Lehrerin) wird seit Sowjetzeiten nicht sehr gut bezahlt und erscheint daher Männern weniger attraktiv. Das wiederum geht auf patriarchale Stereotype zurück, die in Belarus weit verbreitet sind und die in Männern den Ernährer der Familie sehen, die somit keine geringbezahlte Arbeit annehmen oder auf dem »Arbeitsmarkt für Frauen« landen sollten. In der Tat belegt der belarussische "Nationale Bericht zur Umsetzung der Agenda für nachhaltige Entwicklung bis 2030", dass die Löhne und Gehälter von Frauen in Belarus im Durchschnitt 75-80 Prozent derjenigen von Männern betragen. Das erfolgt vor allem aufgrund der Tatsache, dass »Frauen vorwiegend im öffentlichen Bereich beschäftigt sind (beispielsweise in der Bildung, dem Gesundheitswesen, der Kultur, im Sozialbereich), wo die Gehälter relativ gering sind «, mit einem durchschnittlichen Unterschied zwischen Frauenund Männergehältern von 23 Prozent. Auch der belarussische Präsident hat diesen Umstand 2016 in einer Stellungnahme eingeräumt, als er die Schaffung von Bedingungen einforderte, die für eine Statuserhöhung des Lehrerberufs sorgen. 
Einige belarussische Journalisten waren auch der Ansicht, dass Frauen "traditionell zu Konformismus neigen" und keinen Streit suchen, was sie zu idealen Helfern bei Wahlfälschungen mache. Belarussische politische Aktivisten haben belarussischen Lehrern (de facto: Lehrerinnen ) Vorwürfe wegen der Wahlfälschungen gemacht. Der Anführer der »Jungen Front«, Smizer Daschkewitsch, hat sie als "Lehrer der Lüge« bezeichnet, nachdem er einer der Lehrerinnen begegnet war, die zugab, dass sie genötigt wurde, im Wahlkampf für die Präsidentschaftswahlen 2015 an einer Demonstration für den Amtsinhaber teilzunehmen. Belarussische Politikerinnen glauben ebenfalls, dass Frauen »leichter zu manipulieren sind", wie sie sich 2018 in einem Interview für "Radio Free Europe/Radio Liberty« äußerten.

Lidsija Jarmoschyna räumte 2015 während des Präsidentschaftswahlkampfs ein, dass der Frauenanteil in den territorialen Wahlkommissionen 59 Prozent erreichte, während in den Wahlkommissionen auf Stimmbezirksebene 70 Prozent Frauen saßen. Bezeichnenderweise spielt der Belarussische Frauenverband eine systematisch wichtige Rolle im Wahlprozess. Der Verband stellt darüber hinaus ehemalige weibliche Funktionäre ein, die in staatlichen Behörden ihren Posten verloren hatten. So waren mit Stand vom Februar 2018 sowohl die ehemalige Ministerin für das Fernsprech- und Informationswesen, Lilija Ananitsch, als auch die frühere Chefin der Belarussischen Nationalbank, Nadseja Jermakowa, stellvertretende Vorsitzende des Frauenverbandes.

Bei den Wahlen von 2015 wurden 119 Mitglieder des Frauenverbandes als Mitglieder von Wahlkommissionen der verschiedenen Ebenen berufen, wodurch diese Organisation bis zu 6,9 Prozent der Kommissionsmitglieder stellte. Hinzu kam, dass 26 von 422 Anwärtern für die einheimische Wahlbeobachtung vom Belarussischen Frauenverband kamen. In einer Stellungnahme erklärte die ehemalige Nationalbankchefin Nadseja Jermakowa, seinerzeit noch Vorsitzende des Frauenverbandes, frei heraus, dass der Verband bei den Präsidentschaftswahlen 2015 Aljaksandr Lukaschenka unterstützen werde.

Auch die Nachfolgerin von Jermakowa als Verbandsvorsitzende hielt mit ihrer Haltung hinsichtlich politischer Aktivitäten der Mitglieder nicht hinter dem Berg. Die derzeitige Vorsitzende des Frauenverbandes und stellvertretende Vorsitzende des Rates der Republik, Maryjanna Schtschotkina, die früher Ministerin für Arbeit und soziale Sicherung sowie Leiterin des Wahlkampfstabes von Aljaksandr Lukaschenka gewesen war, erklärte in ihrer Rede vor dem nationalen Forum »Familie 21« im Dezember 2017, dass sie bei den Kommunalwahlen eine starke Aktivität von Frauen erwarte.

In der Tat übernahmen Frauen bei der Bildung der Wahlkommissionen für die Kommunalwahlen 2018 eine aktive Rolle und stellten 73,58 Prozent aller Kommissionsmitglieder. Traditionellerweise war der Frauenverband (neben "Belaja Rus«, dem Belarussischen Jugendverband, dem Belarussischen Veteranenverband und dem Belarussischen Gewerkschaftsbund) eine der führenden gesellschaftlichen Vereinigungen, die viele ihrer Mitglieder für die Wahlkommissionen nominiert haben. Sie waren beispielsweise stark in den territorialen Wahlkommissionen (TWK) vertreten, nämlich mit 3.678 Kommissionsmitgliedern; das entspricht 97,3 Prozent der von ihnen nominierten Anwärter. In den Wahlkreiskommissionen waren sie mit 1.871 Kommissionsmitglieder vertreten (95,5\% der nominierten Anwärter), und in den Wahlkommissionen auf Stimmbezirksebene wurden 97 Prozent der nominierten Anwärter in die Kommissionen berufen. Diese Spitzenverbände stellen insgesamt 37,59 Prozent aller Kommissionsmitglieder auf Stimmbezirksebene. Vertreterinnen des Frauenverbandes waren genau wie Mitglieder anderer staatlich geförderter gesellschaftlicher Vereinigungen auch als Wahlbeobachterinnen tätig.

Der Frauenanteil bei den Kommissionsmitgliedern lag auf Stimmbezirksebene bei 72,58 Prozent. In den territorialen Wahlkommissionen betrug er nur 41 Prozent.

Darüber hinaus zeigen die Statistiken, dass in Belarus Frauen bei Kommunalwahlen sehr viel aktiver teilnehmen und zur Wahl gehen als Männer. In Interviews für "Radio Free Europe / Radio Liberty« erklärten Politikerinnen, die bei den Kommunalwahlen 2018 kandidierten, dass sie bei potentiellen Wählern keinen Unterschied in der Haltung ihnen gegenüber gespürt haben, der auf ihr weibliches Geschlecht zurückzuführen wäre. Gleichzeitig erklärten andere politisch aktive Belarussinnen gegenüber dem gleichen Medium, dass Frauen in Belarus keine aktive Haltung und kein Interesse am politischen Geschehen zeigten. Die Teilnahme von Frauen an Wahlen beschränke sich auf ihre Rolle als Mitglieder von Wahlkommissionen

Oppositionelle Aktivistinnen haben vor den Präsidentschaftswahlen 2015 die Kampagne "Frauen gegen Fälschung « ins Leben gerufen, um dem Problem zu begegnen, dass Frauen bei den Wahlen an Fälschungen beteiligt sind. Die Aktivistinnen gehen davon aus, dass Männer sich hier oft hinter "schwachen« Frauen verstecken, weil Fälschungen heftige Strafen nach sich ziehen können. Eine solche Kampagne dürfte in Belarus sehr wohl notwendig sein.

\section{Frauen in belarussischen Staatsorganen}

Der belarussische »Nationale Bericht zur Umsetzung der Agenda für nachhaltige Entwicklung bis 2030 « erwähnt, dass 70,1 Prozent der Staatsangestellten und 68 Prozent der Mitarbeiter der Justiz Frauen sind. Maryjanna 
Schtschotkina sagte im März 2016 in einem Interview für die staatliche Nachrichtenagentur BelTA, dass Belarus versuche, die Vorgaben seines Nationalen Plans zur Gendergleichheit für 2011-2015 zu erfüllen. Die Vorsitzende des Belarussischen Frauenverbandes erklärte, dass der Frauenanteil bei Angestellten in der Exekutive, Legislative und Judikative 55,4 Prozent beträgt. Darüber hinaus überwiegen die weiblichen Angestellten in der lokalen Selbstverwaltung (ohne die dörflichen Exekutivräte) mit einem Anteil von 62,7 Prozent.

Maryjanna Schtschotkina nannte in ihrer Rede vom Dezember 2017 Daten aus dem Bericht und betonte den Umstand, die in den beiden Kammern der Nationalversammlung (des Parlaments), dem Rat der Republik und dem Repräsentantenhaus, die Zahl der Frauen stetig zunimmt. So seien 2016 bei den Parlamentswahlen 33,7 Prozent der gewählten Abgeordneten der beiden Kammern Frauen gewesen, während dieser Anteil 2012 noch bei 29,7 Prozent gelegen hatte. Darüber hinaus waren vor den diesjährigen Kommunalwahlen 46,3 Prozent der Abgeordneten Frauen und 30 Prozent der lokalen Selbstverwaltungen in Belarus wurden von Frauen geleitet. Smizer Kuchlej zufolge, einem Experten der Community »Nasche mnenije» (dt.: »Unsere Meinung«; <http://nmnby.eu>), sei zu erwarten, dass durch die anstehenden Wahlen der Anteil von Frauen in den Kommunalräten zunehmen werde. Das bedeute wiederum eine "Duplizierung" des Anteils unter den Abgeordneten, die die Regierung und die Verwaltung unterstützen, da die meisten Frauen, die für Kommunalräte kandidieren, für lokale Behörden arbeiten, wo sie auch die Mehrheit der Angestellten stellen. Die Ergebnisse der Kommunalwahlen 2018 haben Kuchlej recht gegeben. Nach den Wahlen sind jetzt 48,2 Prozent der gewählten Abgeordneten Frauen. Es gibt also mit jeder neuen Wahl mehr Frauen, die in den Räten vertreten sind.

Der rasche Anstieg des Frauenanteils im belarussischen Parlament erfüllt die Vorgabe von 30 Prozent, die der belarussische Präsident 2004 formuliert hatte. Ähnliche Quoten werden von UN-Übereinkommen und der internationalen Best Practice verlangt. Mit seinen 33 Prozent weiblicher Abgeordneter hält Belarus einen Spitzenplatz innerhalb der Eurasischen Wirtschaftsunion und liegt nach Angaben der Interparlamentarischen Union (IPU) weltweit auf Platz 29. Diese Zahlen verbessern auf der weltweiten Karte zur Gendergleichheit das Image von Belarus. Das Aktivitätsniveau dieser Frauen ist jedoch fraglich. Sie initiieren nur selten Gesetze, sind nicht in den Medien präsent und melden kaum öffentlich zu Wort. Ausnahmen von dieser Regel sind zwei Frauen, die bei den letzten Parlamentswahlen Abgeordnete des Repräsentantenhauses wurden.
Im Jahr 2016 vertraten zwei der 38 gewählten Abgeordneten des Repräsentantenhauses (des Unterhauses des Parlaments), Alena Anisim und Hanna Kanapazkaja, die Opposition in Belarus. Beide Frauen versuchten eine aktive Politik zu betreiben und meldeten sich während der landesweiten sozialen Proteste im Winter und Frühling 2017 zu Wort. Interessanterweise hatte Hanna Kanapazkaja im gleichen Wahlkreis kandidiert wie die ehemalige Präsidentschaftskandidaten Tazjana Karatkewitsch und das Mandat dennoch erringen können. Es bleiben einige Zweifel hinsichtlich der Zuverlässigkeit der Wahlergebnisse, da internationale Beobachter zahlreiche Verstöße bemerkt haben. Wie dem auch sei, allein der Umstand, dass zum ersten Mal seit der Verfassungsreform von 2004, die alle drei Gewalten betraf, zwei Oppositionspolitiker gewählt wurden, wurde von der internationalen Gemeinschaft begrüßt. Viele männliche Politiker und Experten kamen zu dem Schluss, dass diese Situation für Präsident Lukaschenka günstig sei. Sie meinten, der Präsident habe die Wahl dieser Frauen "erlaubt", weil er Frauen nicht als echte politischen Opponenten wahrnehme.

Ungeachtet aller Erklärungen zur Präsenz weiblicher Führungspersonen in der öffentlichen Verwaltung lässt die belarussische Regierung jedoch eine andere Dynamik erkennen. Jetzt, im Februar 2018 sind in der gegenwärtigen belarussischen Regierung sämtliche stellvertretenden Ministerpräsidenten Männer. Im Präsidium des Ministerrates sitzt derzeit nur eine Frau - Natallja Katschanawa, die Leiterin der Präsidialadministration. Bemerkenswerterweise ist sie die erste Frau auf diesem Posten, nachdem sie zuvor als erste Frau den Posten eines stellvertretenden Ministerpräsidenten innegehabt hatte. Allerdings wird jetzt, im Februar 2018, nur eines der derzeit 24 Ministerien, das Ministerium für Arbeit und soziale Sicherung, von einer Frau geleitet, nämlich von Iryna Kaszewitsch. Zusammenfassend lässt sich feststellen, dass die meisten Schlüsselpositionen in der belarussischen Regierung von Männern besetzt sind, ungeachtet aller Erklärungen zur Entwicklung der Genderpolitik in Belarus.

Frauen, die Staatsorgane repräsentieren, geraten oft in die Schlagzeilen oppositioneller Medien. Das Privatleben oder Auftritte dieser Politikerinnen erfahren oft eine besondere mediale Aufmerksamkeit, insbesondere von Seiten oppositioneller Medien. Als der Sohn von Lidsija Jarmoschina 2017 starb, berichteten viele Internetmedien darüber und gaben den Lesern die Möglichkeit, Kommentare hierzu abzugeben, wobei negative Beiträge zum Teil unredigiert blieben...

Auch der Kongress der Gesellschaftlichen Vereinigung "Belaja Rus" am 19. Januar 2018 wurde im belarussischen Netz aufmerksam verfolgt. Da viele Staats- 
funktionäre Mitglieder von »Belaja Rus«sind, kamen in Minsk Offizielle aus ganz Belarus zusammen. Die oppositionelle belarussische Zeitung "Nascha Niwa« brachte mehrere Artikel mit Fotos und bespöttelte besonders weibliche Kongressdelegierte, wobei deren Frisuren und Outfits thematisiert wurden. Gleichzeitig trauten sich die Medien nicht, ähnliche Artikel über männliche Delegierte zu bringen und deren Auftreten zu thematisieren, da sie die - ganz wie eine Mehrheit in der belarussischen Gesellschaft - diese Delegierten als reale Repräsentanten der Macht wahrnahmen, die sie nicht angehen sollten.

\section{Der Präsident und seine weiblichen Vertrauenspersonen}

In den 23 Jahren seiner Präsidentschaft hat Aljaksandr Lukaschenka eine recht einmalige Struktur weiblicher Mitarbeiterinnen geschaffen, die nicht nur bei offiziellen Maßnahmen eingesetzt werden, sondern auch bei inoffiziellen.

Als der belarussische Präsident 1994 sein Amt antrat, war er verheiratet, doch wurde seine Ehefrau nie zu einem wirklichen Teil seines öffentlichen Lebens. Halina Lukaschenka blieb in der kleinen Stadt, in der Aljaksandr Lukaschenka gelebt hatte bevor er Abgeordneter des Obersten Sowjets von Belarus wurde (so hieß das Parlament vor den Verfassungsreformen unter Lukaschenka). Die Schwiegermutter des Präsidenten erklärte 2014 in einem Interview, dass die Ehe ihrer Tochter Halina stets glücklich gewesen sei und sie den Präsidenten immer noch liebe.

Der Präsident enthüllte 2008 die Existenz seines unehelichen Sohnes Mikalaj, der seither bei allen Auslandsbesuchen und wichtigen Ereignissen im Lande selbst zum Begleiter des Präsidenten wurde und somit in dieser Rolle gleichsam die First Lady ersetzte. Lukaschenka selbst erklärte 2009, sein Sohn sei "von Gott«. Der Name von Mikalajs Mutter ist nie öffentlich bekannt gegeben worden, doch glauben viele Belarussen, dass es sich um Iryna Abalskaja handelt, die persönliche Ärztin des Präsidenten, die nun das angesehenste medizinische Zentrum des Landes leitet. Ljudmila Pastajalka, die Mutter von Iryna Abelskaja, war nach ihrer Ernennung durch Lukaschenka mehrere Jahre belarussische Ministerin für Gesundheit. Iryna Abalskaja hat nie öffentlich zugegeben, dass Mikalaj Lukaschenka ihr Sohn ist, obwohl sie ihn in ihren Interviews gegenüber belarussischen Journalisten als ihren jüngsten Sohn erwähnt hat, allerdings ohne seinen Namen auszusprechen.

Im Mai 2014 gab Lukaschenka dem unabhängigen russischen Fernsehsender "Doschd" ein Interview. Das Gespräch führte die prominente Journalistin Ksenija Sobtschak, die im März 2018 bei den Präsidentschafts- wahlen in Russland kandidiert. Das Interview warf ein Licht auf viele persönliche Details in Lukaschenkas Leben. Er meinte, dass er - auch wenn sie fast dreißig Jahre nicht mehr zusammengelebt hatten - sich nicht von seiner Ehefrau scheiden lassen wolle, weil er nicht "die Mutter seiner Kinder traumatisieren wollte«. Das Geheimnis und die Spekulationen um die aktuelle First Lady haben nie aufgehört und belarussische wie auch ausländische Journalisten versuchen weiterhin Fotos von den Frauen zu machen, die bei offiziellen internationalen oder belarussischen Veranstaltungen an der Seite des Präsidenten in Erscheinung treten.

Aljaksandr Lukaschenka hat die Neigung, sich als jemanden darzustellen, der bei Frauen beliebt ist, und er versucht dieses Image weiter zu pflegen. Im demselben Interview für "Doschd « bestätigte er, dass er bei den unterschiedlichen öffentlichen Anlässen gern in Begleitung von Frauen weilt, und nicht von staatlichen Funktionären. Er räumte auch ein, dass eine seiner neuen Begleiterinnen eine junge Dame namens Darja Schmanaj sei, die offiziell im protokollarischen Dienst des Präsidenten angestellt ist. Berichten zufolge wurde Lukaschenka während eines Schönheitswettbewerbs, den sie gewann, auf sie aufmerksam wurde. Anschließend erhielt sie einen Posten beim protokollarischen Dienst des Präsidenten, wo attraktive, model-hafte junge Frauen bei internationalen Empfängen und öffentlichen Feiern stets als Assistentinnen willkommen sind.

$\mathrm{Zu}$ den anderen Begleiterinnen, mit denen er seine Schwiegermutter oder Sportveranstaltungen besuchte, gehören Fernsehjournalistinnen wie Palina Schuba, Sängerinnen wie Alena Lanskaja (die Belarus beim »Eurovision Song Contest « vertreten hat) und Profisportlerinnen wie Ksenija Sankowitsch. Einige von ihnen wurden sogar bei den letzten Präsidentschaftswahlen 2015 Lukaschenkas Vertrauenspersonen, beispielsweise die Gymnastin Ljubou Tscharkaschyna. Andere machten später politisch Karriere, wie im Fall der Sängerin Iryna Darafejewa, die Abgeordnete des Repräsentantenhauses wurde, nachdem sie inoffiziell als Lieblingssängerin des Präsidenten kolportiert wurde.

Eine der früheren Begleiterinnen Lukaschankas, Natallja Ejsmant machte erfolgreich beim Fernsehen Karriere, bevor sie Pressesprecherin des Präsidenten wurde. Ihr Ehemann Iwan Ejsmant wurde am 6. Februar 2018 zum Leiter der Nationalen staatlichen Fernsehund Rundfunkgesellschaft ernannt. Das ist einer der wichtigsten Posten in der belarussischen Fernsehbranche. Beides ist ein Beleg für die äußerst wichtige Rolle, die die Ejsmants in der staatlichen politischen Hierarchie einnehmen. Interessanterweise ist der Ehemann von Natallja Pjatkewitsch, Ejsmants Vorgängerin als Pressesprecherin Lukaschenkas, vom belarussichen Präsiden- 
ten zum Generaldirektor von "Belarus 24" geworden, des wichtigsten staatlichen Fernsehsenders des Landes. Natallja Pjatkewitsch hingegen stieg zur stellvertretenden Leiterin der Präsidialadministration und zur persönlichen Beraterin des Präsidenten auf.

Die belarussische Öffentlichkeit verfolgt nicht nur aufmerksam das Privatleben des Präsidenten, sondern schenkt auch den Äußerungen Lukaschenkas über Frauen viel Beachtung. Am 9. März 2017 rief Lukaschenka auf einem Treffen mit wichtigen Regierungsvertretern, bei der die Folgen des berüchtigten Erlasses Nr. 3 erörtert wurden (der inoffiziell "Sozialschmarotzererlass" genannt und der landesweit zahlreiche Proteste ausgelöst hatte), die Funktionäre dazu auf dafür zu sorgen, dass ihre "Ehemänner und Ehefrauen, Liebhaber und Liebhaberinnen, Familienangehörigen und Freunde« in Beschäftigung sind. Diese Äußerung sorgte in den sozialen Medien für ironische Kommentare von Internetnutzern, die erklärten, dass sie die Position einer Liebhaberin des Präsidenten wollten, um dadurch eine Anstellung zu bekommen. Später wurden diese Zeilen aus dem Stenogramm auf der offiziellen Website des Präsidenten gestrichen; sie hatten aber in der Bevölkerung für negative Reaktionen gesorgt.

\section{Schlussfolgerungen}

Frauen spielen in Belarus eine sehr spezifische Rolle in den politischen Prozessen und bei der Zusammensetzung der Staatsorgane. Einerseits überwiegen Frauen in Behörden der Exekutive wie auch bei der Organisation und Durchführung eines so wichtigen politischen Prozesses wie den Wahlen. Andererseits haben Frauen in den wichtigsten Regierungsorganen nur selten Führungspositionen inne. Hierfür gibt es drei Gründe.

Erstens funktioniert die belarussische Gesellschaft immer noch im Einklang mit patriarchalen Mustern, ungeachtet des verkündeten Plans zur Gendergleichheit, den der belarussische Staat umzusetzen hat. Der Plan bleibt in etlicher Hinsicht unwirksam und oberflächlich. $\mathrm{Zu}$ den patriarchalen Zügen der belarussischen Gesellschaft gehören nicht nur eine erhebliche Einkommenslücke und das Überwiegen weiblicher Beschäftigter in den geringer bezahlten Bereichen der Wirtschaft, sondern auch Verhaltensmuster wie jene, dass Frauen tendenziell weniger angesehene Beschäftigungen haben, weniger Verantwortung tragen und diese Realität akzeptieren.

Zweitens zeigen die belarussischen Behörden auf höchster Ebene kaum Interesse, weibliche Führungskräfte in höhere Positionen zu bringen. Die meisten strategisch wichtigen Ressorts (Außenministerium, Verteidigung, Justiz, Verfassungsgericht usw.) sind seit der Unabhängigkeit von Belarus immer von Männern geleitet worden. Der belarussische Präsident tendiert dazu, sich mit Frauen zu umgeben, und fördert sie eher aufgrund persönlicher Beziehungen zu ihm und seiner Umgebung, denn aufgrund persönlicher Qualitäten. Allein die Tatsache, dass Aljaksandr Lukaschenka nie öffentlich in Begleitung seiner offiziellen Ehefrau oder einer weiblichen Begleitung in der Funktion als First Lady auftritt, stuft die Rolle weiblicher Partner von Staatschefs öffentlich herab. Dies gilt umso mehr, als weltweit eine gesteigerte Bedeutung dieser Rolle zu beobachten ist.

Schließlich werden Politikerinnen und Funktionärinnen darüber hinaus durch den öffentlichen Diskurs marginalisiert. Hinsichtlich des Potentials belarussischer Frauen für zentrale staatliche Anliegen in Belarus sind Zweifel angebracht. Insgesamt sind sehr viel mehr Reformen vonnöten, um Belarus auf einen Weg zu realer Gendergleichheit zu bringen. Aufgrund der Schwäche der Bewegung für Gendergleichheit im Land dürften hier nur sehr langsame Veränderungen zu erwarten sein.

Übersetzung aus dem Englischen: Hartmut Schröder

\section{Über die Autorin}

Veranika Laputska ist Doktorandin am Graduiertenkolleg der Polnischen Akademie der Wissenschaften in Warschau. Ihre Dissertation befasst sich mit visueller Propaganda im modernen Belarus. Zu ihren Forschungsinteressen gehören Medien- und Propagandaforschung, Demokratisierungsprozesse in Mittel- und Osteuropa, Nationalismus und Nationenbildung sowie Judaistik. 


\section{Global Gender Gap Report 2017 (Bericht des »World Economic Forum«)}

Grafik 1a: Belarus im globalen Ranking: Rang im Vergleich (Auswahl aus 144 Staaten) Globaler Index

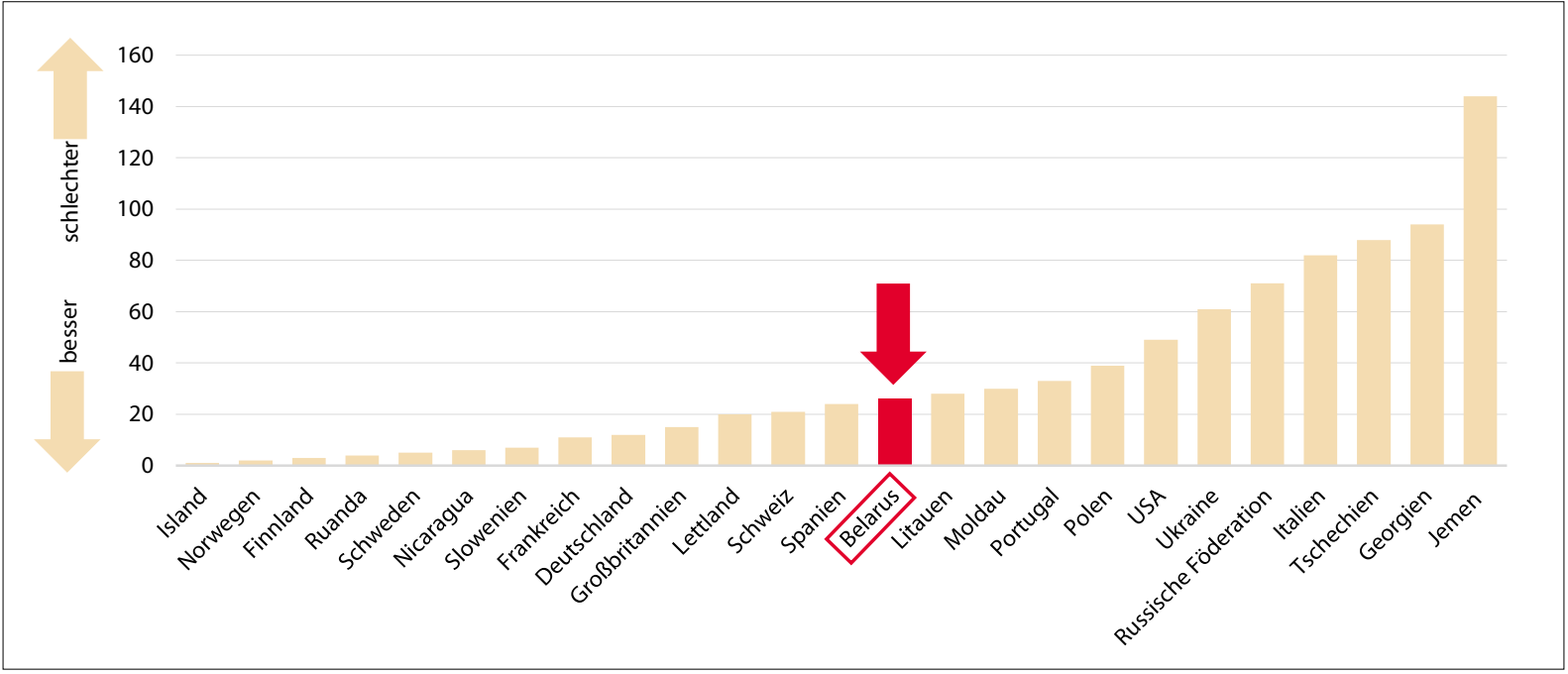

Quelle: Global Gender Gap Report 2017 (World Economic Forum), S. 11-13, <https://www.weforum.org/reports/ the-global-gender-gap-report-2017>

Grafik 1b: Belarus im globalen Ranking: Rang im Vergleich (Auswahl aus 144 Staaten) Wirtschaftliche Teilhabe und Chancen

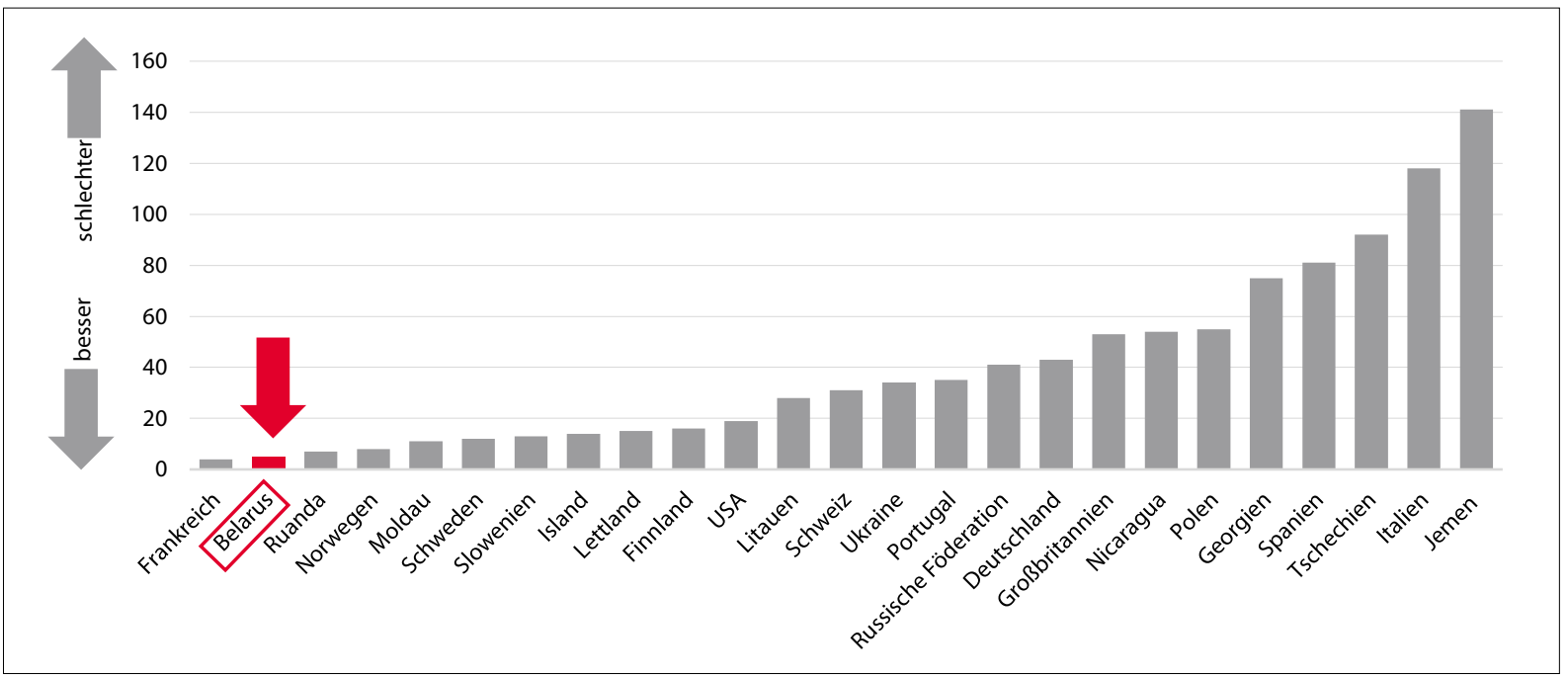

Quelle: Global Gender Gap Report 2017 (World Economic Forum), S. 11-13, <https://www.weforum.org/reports/ the-global-gender-gap-report-2017> 
Grafik 1c: Belarus im globalen Ranking: Rang im Vergleich (Auswahl aus 144 Staaten) Bildungsniveau

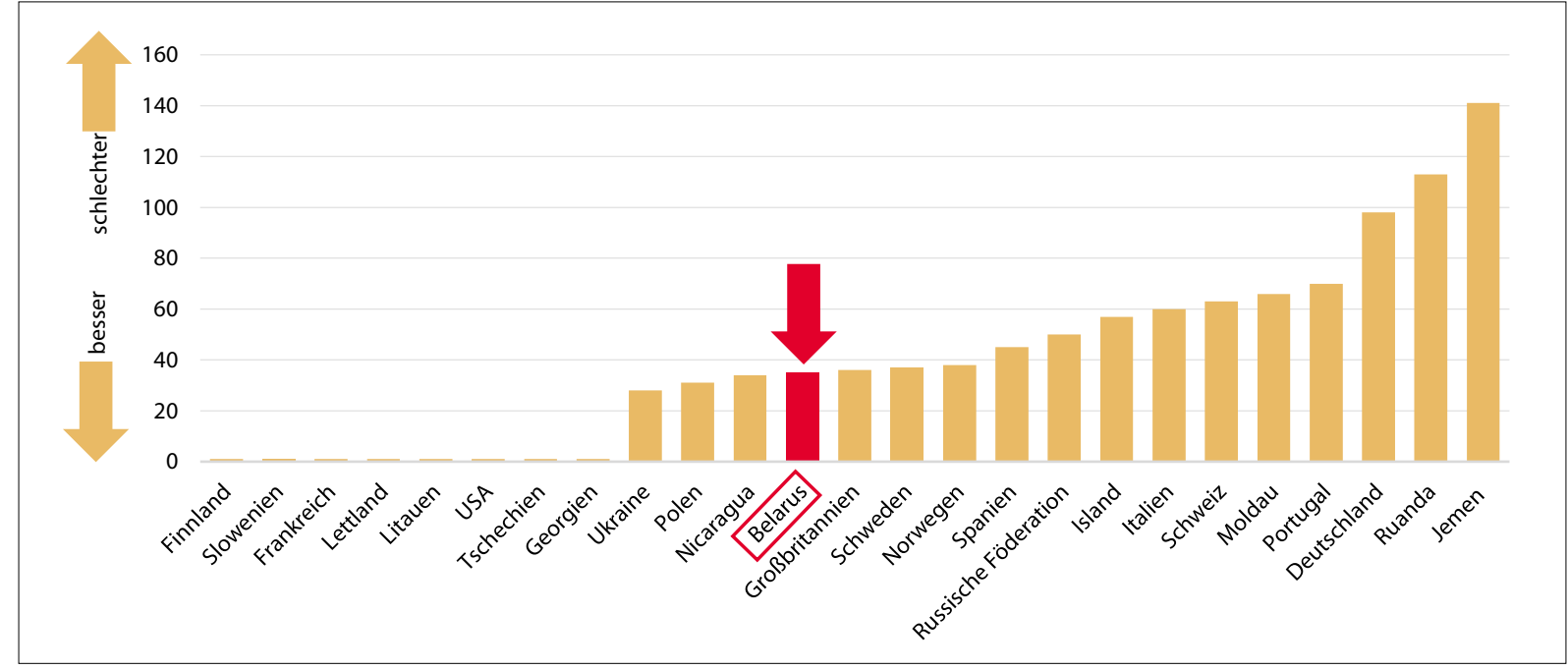

Quelle: Global Gender Gap Report 2017 (World Economic Forum), S. 11-13, <https://www.weforum.org/reports/ the-global-gender-gap-report-2017>

Grafik 1d: Belarus im globalen Ranking: Rang im Vergleich (Auswahl aus 144 Staaten) Gesundheit und Überleben

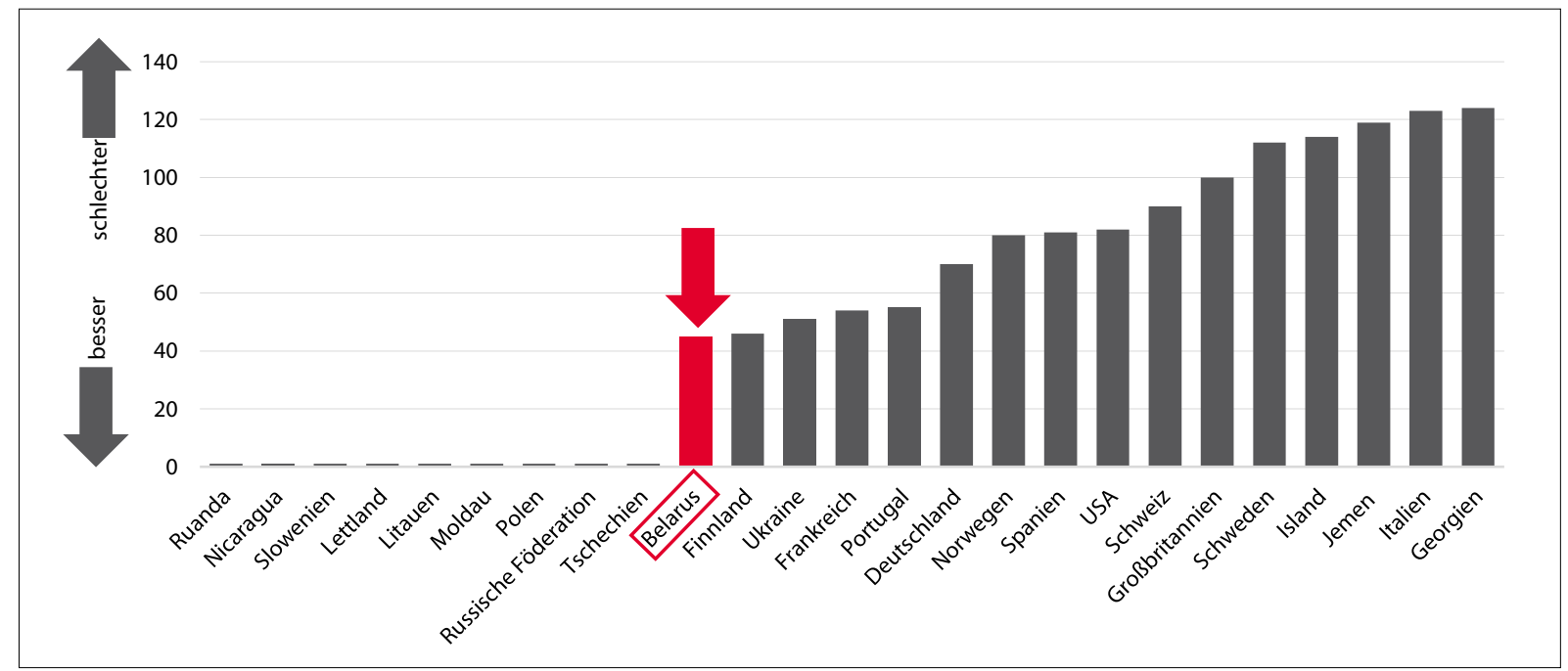

Quelle: Global Gender Gap Report 2017 (World Economic Forum), S. 11-13, <https://www.weforum.org/reports/ the-global-gender-gap-report-2017> 
Grafik 1e: Belarus im globalen Ranking: Rang im Vergleich (Auswahl aus 144 Staaten) Politische Ermächtigung

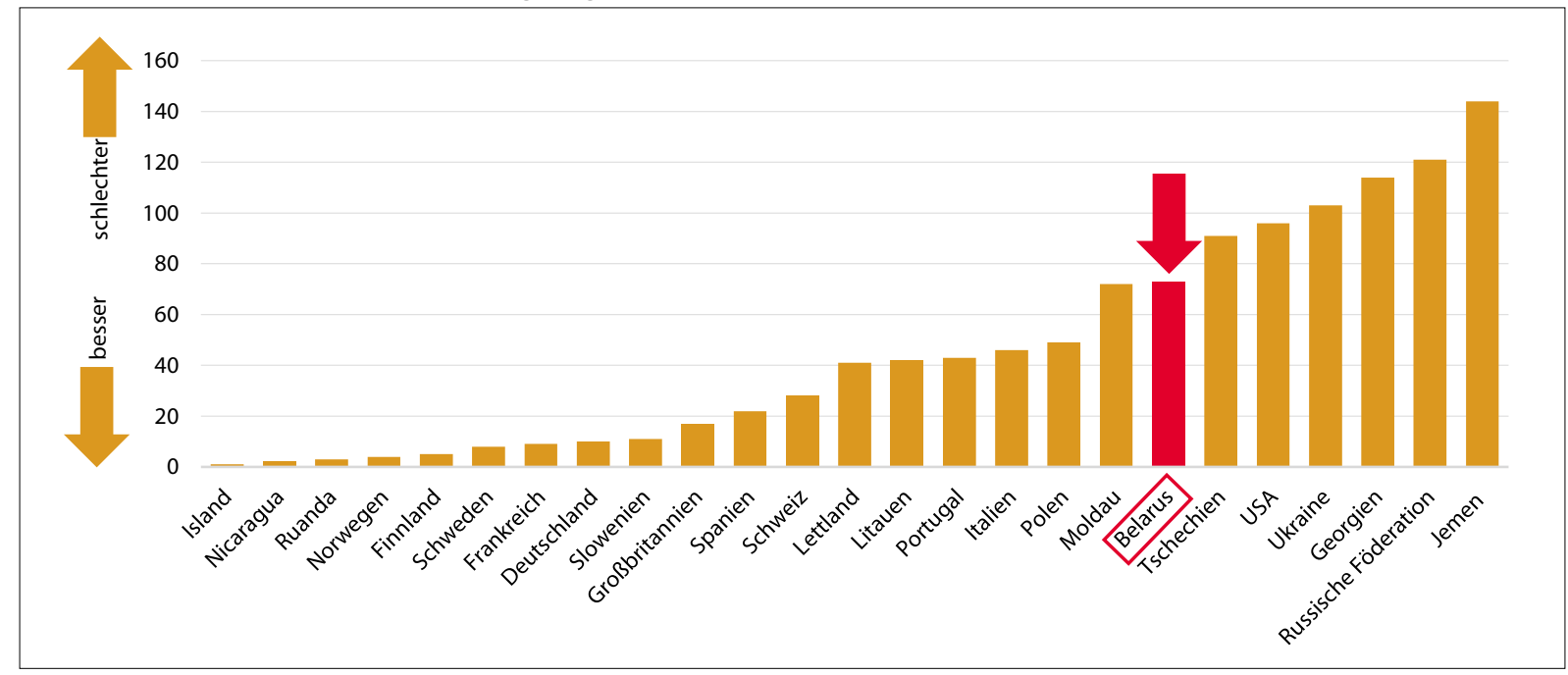

Quelle: Global Gender Gap Report 2017 (World Economic Forum), S. 11-13, <https://www.weforum.org/reports/ the-global-gender-gap-report-2017>

Grafik 2: Belarus im globalen Ranking: Politische Ermächtigung (Platzierung im globalen Vergleich und Frauenanteil in Prozent)

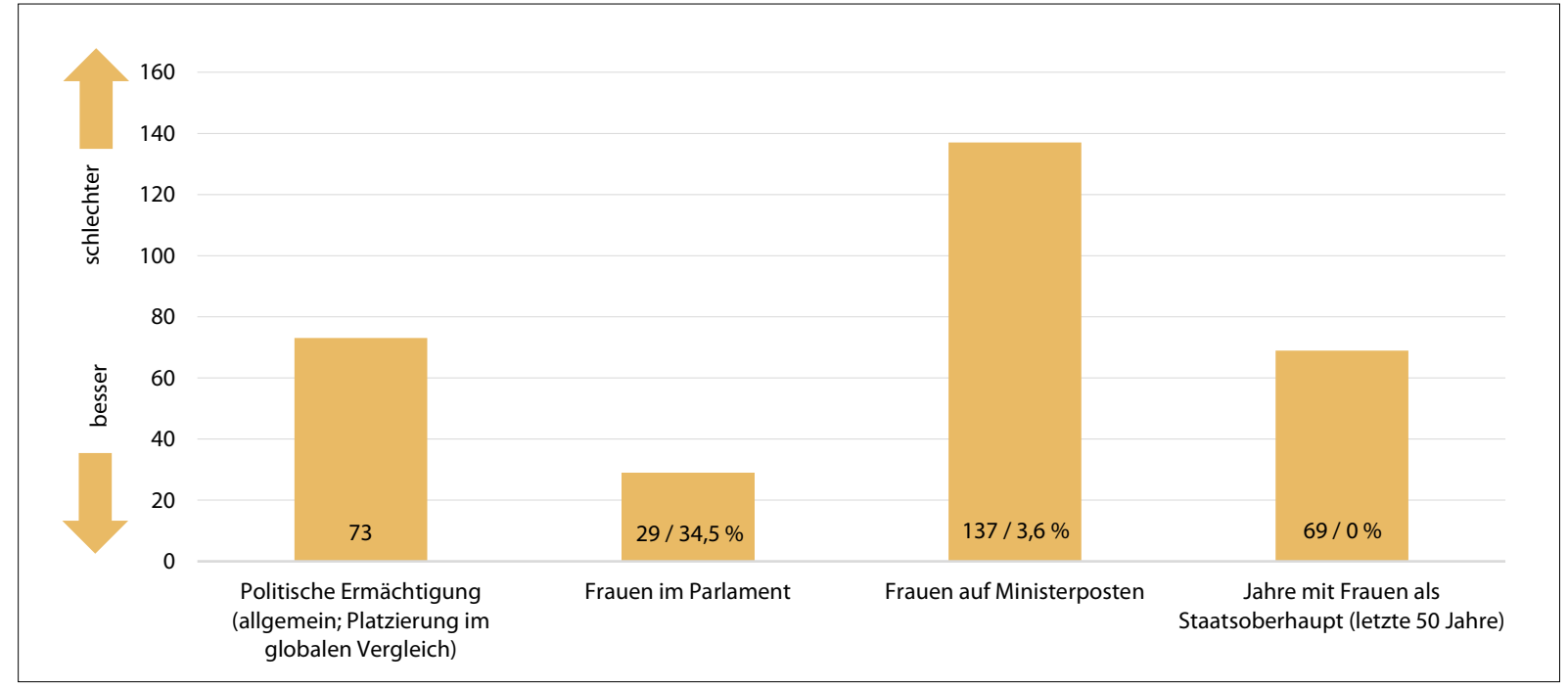

Quelle: Global Gender Gap Report 2017 (World Economic Forum), S. 82, <https://www.weforum.org/reports/the-global -gender-gap-report-2017> 
Tabelle 1: Belarus im globalen Ranking: Rang im Vergleich (Auswahl aus 144 Staaten)

\begin{tabular}{|c|c|c|c|c|c|}
\hline & Globaler Index & $\begin{array}{l}\text { Wirtschaftliche } \\
\text { Teilhabe und } \\
\text { Chancen }\end{array}$ & Bildungsniveau & $\begin{array}{l}\text { Gesundheit und } \\
\text { Überleben }\end{array}$ & $\begin{array}{c}\text { Politische } \\
\text { Ermächtigung }\end{array}$ \\
\hline Island & 1 & 14 & 57 & 114 & 1 \\
\hline Norwegen & 2 & 8 & 38 & 80 & 4 \\
\hline Finnland & 3 & 16 & 1 & 46 & 5 \\
\hline Ruanda & 4 & 7 & 113 & 1 & 3 \\
\hline Schweden & 5 & 12 & 37 & 112 & 8 \\
\hline Nicaragua & 6 & 54 & 34 & 1 & 2 \\
\hline Slowenien & 7 & 13 & 1 & 1 & 11 \\
\hline Frankreich & 11 & 4 & 1 & 54 & 9 \\
\hline Deutschland & 12 & 43 & 98 & 70 & 10 \\
\hline Großbritannien & 15 & 53 & 36 & 100 & 17 \\
\hline Lettland & 20 & 15 & 1 & 1 & 41 \\
\hline Schweiz & 21 & 31 & 63 & 90 & 28 \\
\hline Spanien & 24 & 81 & 45 & 81 & 22 \\
\hline Belarus & 26 & 5 & 35 & 45 & 73 \\
\hline Litauen & 28 & 28 & 1 & 1 & 42 \\
\hline Moldau & 30 & 11 & 66 & 1 & 72 \\
\hline Portugal & 33 & 35 & 70 & 55 & 43 \\
\hline Polen & 39 & 55 & 31 & 1 & 49 \\
\hline USA & 49 & 19 & 1 & 82 & 96 \\
\hline Ukraine & 61 & 34 & 28 & 51 & 103 \\
\hline Russische Föderation & 71 & 41 & 50 & 1 & 121 \\
\hline Italien & 82 & 118 & 60 & 123 & 46 \\
\hline Tschechien & 88 & 92 & 1 & 1 & 91 \\
\hline Georgien & 94 & 75 & 1 & 124 & 114 \\
\hline Jemen & 144 & 141 & 141 & 119 & 144 \\
\hline
\end{tabular}

Quelle: Global Gender Gap Report 2017 (World Economic Forum), S. 11-13, <https://www.weforum.org/reports/ the-global-gender-gap-report-2017> 


\section{Human Development Report 2016 (Bericht von UNDP)}

Grafik 3: Index der Gender-Ungleichheit in Belarus im Vergleich (2015; Auswahl aus 188 Staaten)

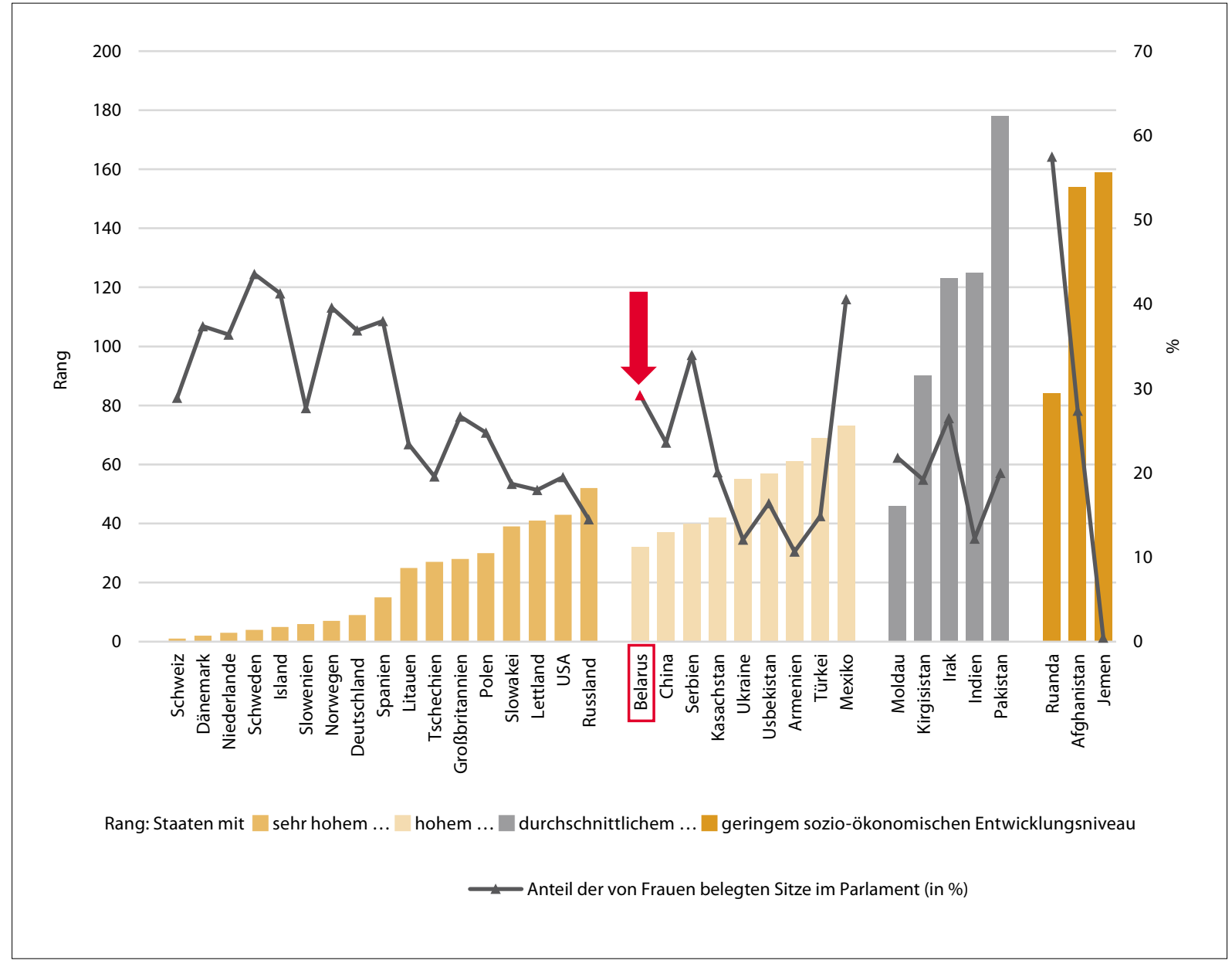

Quelle: Human Development Report 2016 (UNDP), S. 214-217, <http://hdr.undp.org/sites/default/files/2016_human_develop ment_report.pdf>

Tabelle 2: Index der Gender-Ungleichheit in Belarus im Vergleich (2015; Auswahl aus 188 Staaten)

\begin{tabular}{|l|c|c|}
\hline & Rang & $\begin{array}{c}\text { Anteil der von Frauen belegten Sitze } \\
\text { im Parlament (in \%) }\end{array}$ \\
\hline Staaten mit sehr hohem sozio-ökonomischen Entwicklungsniveau \\
\hline Schweiz & 1 & 28,9 \\
\hline Dänemark & 2 & 37,4 \\
\hline Niederlande & 3 & 36,4 \\
\hline Schweden & 4 & 43,6 \\
\hline Island & 5 & 41,3 \\
\hline Slowenien & 6 & 27,7 \\
\hline
\end{tabular}


Tabelle 2: Index der Gender-Ungleichheit in Belarus im Vergleich (2015; Auswahl aus 188 Staaten) (Fortsetzung)

\begin{tabular}{|c|c|c|}
\hline & Rang & $\begin{array}{c}\text { Anteil der von Frauen belegten Sitze } \\
\text { im Parlament (in \%) }\end{array}$ \\
\hline Norwegen & 7 & 39,6 \\
\hline Deutschland & 9 & 36,9 \\
\hline Spanien & 15 & 38,0 \\
\hline Litauen & 25 & 23,4 \\
\hline Tschechien & 27 & 19,6 \\
\hline Großbritannien & 28 & 26,7 \\
\hline Polen & 30 & 24,8 \\
\hline Slowakei & 39 & 18,7 \\
\hline Lettland & 41 & 18,0 \\
\hline USA & 43 & 19,5 \\
\hline Russland & 52 & 14,5 \\
\hline \multicolumn{3}{|c|}{ Staaten mit hohem sozio-ökonomischen Entwicklungsniveau } \\
\hline Belarus & 32 & 29,2 \\
\hline China & 37 & 23,6 \\
\hline Serbien & 40 & 34,0 \\
\hline Kasachstan & 42 & 20,1 \\
\hline Ukraine & 55 & 12,1 \\
\hline Usbekistan & 57 & 16,4 \\
\hline Armenien & 61 & 10,7 \\
\hline Türkei & 69 & 14,9 \\
\hline Mexiko & 73 & 40,6 \\
\hline \multicolumn{3}{|c|}{ Staaten mit durchschnittlichem sozio-ökonomischen Entwicklungsniveau } \\
\hline Moldau & 46 & 21,8 \\
\hline Kirgisistan & 90 & 19,2 \\
\hline Irak & 123 & 26,5 \\
\hline Indien & 125 & 12,2 \\
\hline Pakistan & 178 & 20,0 \\
\hline \multicolumn{3}{|c|}{ Staaten mit geringem sozio-ökonomischen Entwicklungsniveau } \\
\hline Ruanda & 84 & 57,5 \\
\hline Afghanistan & 154 & 27,4 \\
\hline Jemen & 159 & 0,5 \\
\hline
\end{tabular}

Quelle: Human Development Report 2016 (UNDP), S. 214-217, <http://hdr.undp.org/sites/default/files/2016_human_develop ment_report.pdf> 


\section{Frauen und Männer in der Republik Belarus, 2016 (Bericht des Nationalen Komitees für Statistik, Belstat)}

\section{Grafik 4: Frauen im Parlament}

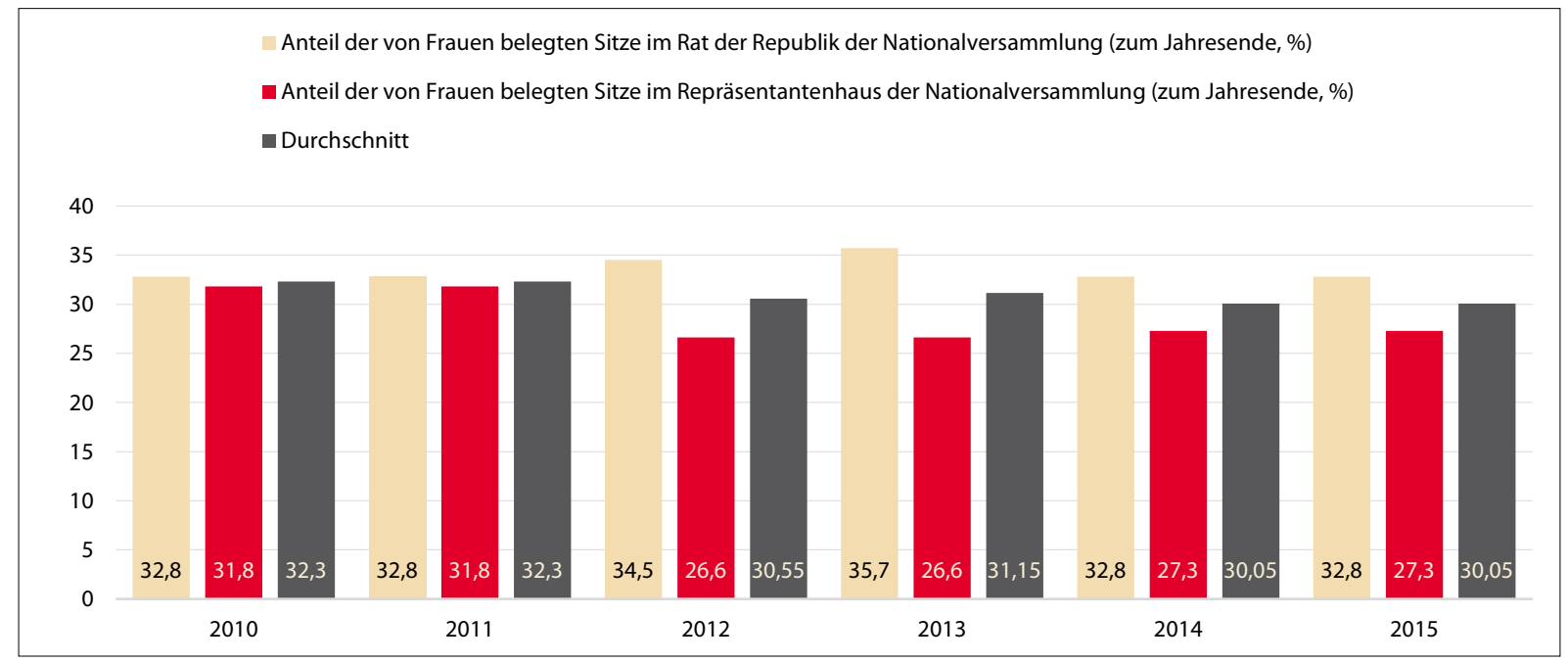

Quelle: Women and Men in The Republic of Belarus, 2016, Nationales Komitee für Statistik der Republik Belarus (Belstat), S. 18, $<$ http://www.belstat.gov.by/ofitsialnaya-statistika/publications/izdania/public_compilation/index_5079/>

Tabelle 4: Anteil der Frauen und Männer im Parlament nach Alter (zum 1. Januar)

\begin{tabular}{|c|c|c|c|c|c|c|c|c|c|c|c|c|}
\hline & \multicolumn{2}{|c|}{2010} & \multicolumn{2}{|c|}{2011} & \multicolumn{2}{|c|}{2012} & \multicolumn{2}{|c|}{2013} & \multicolumn{2}{|c|}{2014} & \multicolumn{2}{|c|}{2015} \\
\hline & $\begin{array}{c}\text { Frau- } \\
\text { en }\end{array}$ & $\begin{array}{c}\text { Män- } \\
\text { ner }\end{array}$ & $\begin{array}{c}\text { Frau- } \\
\text { en }\end{array}$ & $\begin{array}{c}\text { Män- } \\
\text { ner }\end{array}$ & $\begin{array}{c}\text { Frau- } \\
\text { en }\end{array}$ & $\begin{array}{c}\text { Män- } \\
\text { ner }\end{array}$ & $\begin{array}{c}\text { Frau- } \\
\text { en }\end{array}$ & $\begin{array}{c}\text { Män- } \\
\text { ner }\end{array}$ & $\begin{array}{c}\text { Frau- } \\
\text { en }\end{array}$ & $\begin{array}{c}\text { Män- } \\
\text { ner }\end{array}$ & $\begin{array}{c}\text { Frau- } \\
\text { en }\end{array}$ & $\begin{array}{c}\text { Män- } \\
\text { ner }\end{array}$ \\
\hline \multicolumn{13}{|c|}{ Im Rat der Republik } \\
\hline Insgesamt & 19 & 39 & 19 & 39 & 19 & 39 & 20 & 38 & 20 & 36 & 19 & 39 \\
\hline \multicolumn{13}{|c|}{ davon im Alter von: } \\
\hline 30-39 Jahren & 1 & 3 & 1 & 3 & 1 & 3 & 1 & 3 & 1 & 1 & 1 & 1 \\
\hline 40-49 Jahren & 8 & 2 & 5 & 3 & 4 & 1 & 6 & 7 & 6 & 8 & 5 & 8 \\
\hline 50-59 Jahren & 10 & 20 & 11 & 17 & 12 & 18 & 13 & 16 & 12 & 14 & 12 & 17 \\
\hline über 60 Jahren & 0 & 14 & 2 & 16 & 2 & 17 & 0 & 12 & 1 & 13 & 1 & 13 \\
\hline \multicolumn{13}{|c|}{ Im Repräsentantenhaus } \\
\hline Insgesamt & 35 & 75 & 35 & 75 & 35 & 75 & 29 & 80 & 29 & 80 & 30 & 80 \\
\hline \multicolumn{13}{|c|}{ davon im Alter von: } \\
\hline 30-39 Jahren & 2 & 4 & 2 & 4 & 2 & 3 & 2 & 7 & 1 & 5 & 1 & 5 \\
\hline 40-49 Jahren & 8 & 11 & 7 & 10 & 4 & 9 & 11 & 10 & 9 & 8 & 10 & 7 \\
\hline 50-59 Jahren & 22 & 42 & 22 & 39 & 23 & 37 & 16 & 52 & 18 & 51 & 17 & 46 \\
\hline über 60 Jahren & 3 & 18 & 4 & 22 & 6 & 26 & 0 & 11 & 1 & 16 & 2 & 22 \\
\hline
\end{tabular}

Quelle: Women and Men in The Republic of Belarus, 2016, Nationales Komitee für Statistik der Republik Belarus (Belstat), S. 129, $<$ http://www.belstat.gov.by/ofitsialnaya-statistika/publications/izdania/public_compilation/index_5079/> 
Tabelle 5: Anteil der Angestellten staatlicher Organisationen in Belarus nach Positionen (zum 1. Juli 2015; \%)

\begin{tabular}{|l|c|c|c|c|}
\hline \multirow{2}{*}{} & \multirow{2}{*}{ Frauen } & \multirow{2}{*}{ Männer } & \multicolumn{2}{c|}{ Nach Geschlecht: } \\
\cline { 3 - 5 } & & & Frauen & Männer \\
\hline Angestellte insgesamt & 100 & 100 & 70,1 & 29,9 \\
\hline davon: & \multicolumn{2}{|l}{} \\
\hline $\begin{array}{l}\text { Organisationsleiter und ihre } \\
\text { Stellvertreter }\end{array}$ & 10,4 & 20,2 & 54,7 & 45,3 \\
\hline Abteilungsleiter und ihre Stellvertreter & 24,8 & 30 & 65,9 & 34,1 \\
\hline Assistenz der Leitung, Berater & 2,9 & 3 & 68,9 & 31,1 \\
\hline Leitende Fachkraft & 40,7 & 25,1 & 79,2 & 20,8 \\
\hline Gehobene Fachkraft & 6,7 & 2,6 & 85,7 & 14,3 \\
\hline Andere Fachkräfte & 8,9 & 11,3 & 64,8 & 35,2 \\
\hline Juristische Mitarbeiter & 5,5 & 7,5 & 63,3 & 36,7 \\
\hline Andere Mitarbeiter & 0,1 & 0,3 & 43,7 & 56,3 \\
\hline
\end{tabular}

Quelle: Women and Men in The Republic of Belarus, 2016, Nationales Komitee für Statistik der Republik Belarus (Belstat), S. 130, $<$ http://www.belstat.gov.by/ofitsialnaya-statistika/publications/izdania/public_compilation/index_5079/>

Tabelle 6: Anteil der Angestellten staatlicher Organisationen in Belarus nach Ausbildung (zum 1. Juli 2015; \%)

\begin{tabular}{|l|c|c|c|c|}
\hline \multirow{2}{*}{} & \multirow{2}{*}{ Frauen } & \multirow{2}{*}{ Männer } & \multicolumn{2}{|c|}{ Nach Geschlecht: } \\
\cline { 3 - 5 } & & & Frauen & Männer \\
\hline Angestellte insgesamt & 100 & 100 & 70,1 & 29,9 \\
\hline davon mit abgeschlossener Ausbildung: & \multicolumn{5}{|l}{} \\
\hline Hochschulabschluss & 92,2 & 96,6 & 69,1 & 30,9 \\
\hline Berufsausbildung & 7,1 & 2,8 & 85,6 & 14,4 \\
\hline keins von den beiden & 0,7 & 0,6 & 73 & 27 \\
\hline
\end{tabular}

Quelle: Women and Men in The Republic of Belarus, 2016, Nationales Komitee für Statistik der Republik Belarus (Belstat), S. 132, $<$ http://www.belstat.gov.by/ofitsialnaya-statistika/publications/izdania/public_compilation/index_5079/>

Tabelle 7: Anteil der Angestellten staatlicher Organisationen in Belarus nach Berufserfahrung in staatlichen Strukturen (zum 1. Juli 2015; \%)

\begin{tabular}{|l|c|c|c|c|}
\hline \multirow{2}{*}{} & Frauen & Männer & \multicolumn{2}{c|}{ Nach Geschlecht: } \\
\cline { 2 - 5 } & & & Frauen & Männer \\
\hline Angestellte insgesamt & 100 & 100 & 70,1 & 29,9 \\
\hline davon mit Erfahrung in staatlichen Strukturen: & 5,9 & 5,6 & 71 & 29 \\
\hline unter 1 Jahr & 10,6 & 9,1 & 73,3 & 26,7 \\
\hline 1-2 Jahre & 21,3 & 19,9 & 71,5 & 28,5 \\
\hline 3-7 Jahre & 22,6 & 24,7 & 68,2 & 31,8 \\
\hline 8-14 Jahre & 16,6 & 17,5 & 68,9 & 31,1 \\
\hline 15-19 Jahre & 13,5 & 14,7 & 68,3 & 31,7 \\
\hline 20-24 Jahre & 9,5 & 8,5 & 72,3 & 27,7 \\
\hline 25 Jahre und mehr & & \multicolumn{4}{|l}{} \\
\hline
\end{tabular}

Quelle: Women and Men in The Republic of Belarus, 2016, Nationales Komitee für Statistik der Republik Belarus (Belstat), S. 132, $<$ http://www.belstat.gov.by/ofitsialnaya-statistika/publications/izdania/public_compilation/index_5079/> 


\section{Frauen in den Wahlkommissionen, gewählten Staatsorganen und Ministerien}

\section{Grafik 5: Anteil der Frauen in den Wahlkommissionen bei den Kommunalwahlen (\%)}

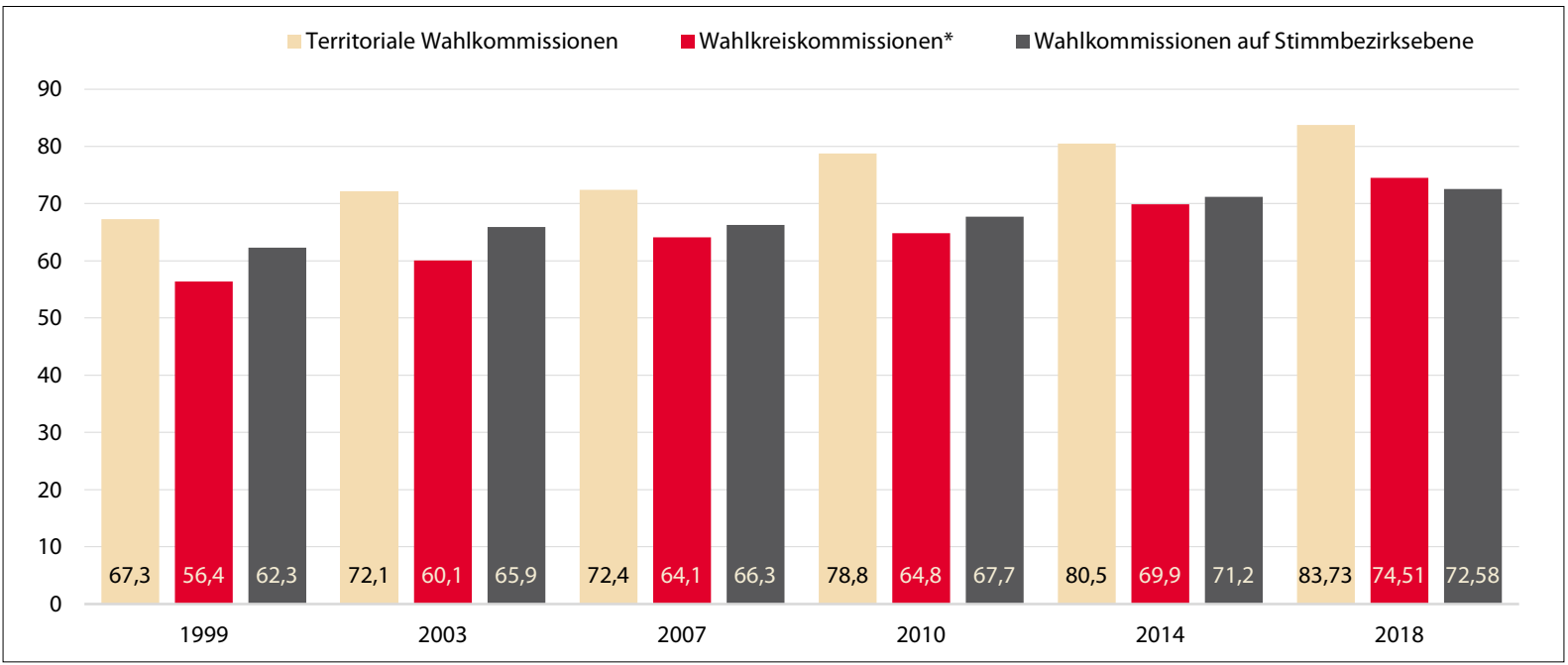

* In Minsk: Territoriale Wablkommissionen, die als Wablkreiskommissionen funktionieren

Quellen: Wadsim Bylina: Sklad wybartschych kamisij: Asnounyja tendenzyi wa umowach autarytarnaj sistemy Belarusi (mjaszowyja wybary, 1999-2014 gady); in: The Fourth International Congress of Belarusian Studies, Working Papers, Volume 4 (2015), S. 57, <http:// icbs.pali tyka.org/wp-content/uploads/2014/papers/02-02_Bilina.pdf>; Zentrale Wablkommission der Republik Belarus: Swedenija o sostawe utschastkowyh izbiratelnyh kommissij po wyboram w mestnye Sowety deputatow dwadtsat wosmogo sosywa, <http://rec.gov.by/ sites/default/files/pdf/Elections-MS28-elect_30-31.pdf>; Zentrale Wahlkommission der Republik Belarus: Swedenija o sostawe terrotorialnyh izbiratelnyh kommissij po wyboram w mestnye Sowety deputatow dwadtsat wosmogo sosywa, <http://rec.gov.by/sites/default/ files/pdf/Elections-MS28-elect_3-4.pdf>; Zentrale Wahlkommission der Republik Belarus: Swedenija o sostawe okruzhnyh, terrotorialnyh w rajonah g. Minska izbiratelnyh kommissij po wyboram w mestnye Sowety deputatow dwadtsat wosmogo sosywa, <http://rec. gov.by/sites/default/files/pdf/Elections-MS28-elect_12-13.pdf>

Grafik 6: Anteil der Frauen in den Wahlkommissionen während der Parlamentswahlen $(2016, \%)$

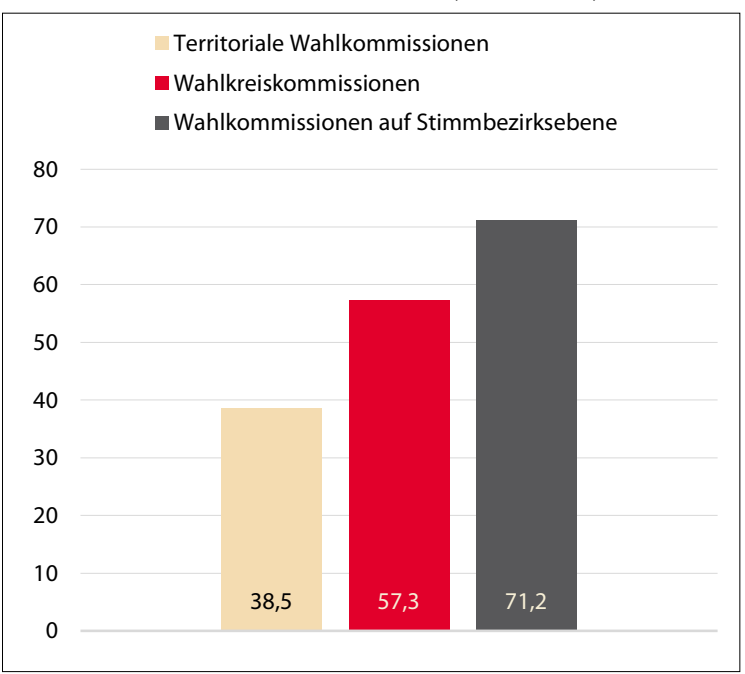

Quelle: OSCE/ODIHR Election Observation Mission Final Report, Parliamentary Elections, 11 September 2016, Republic of Belarus, S. 7, <https://www.osce.org/odihr/ elections/287486?download=true $>$
Grafik 7: Anteil der Frauen in den Wahlkommissionen während der Präsidentschaftswahlen $(2015, \%)$

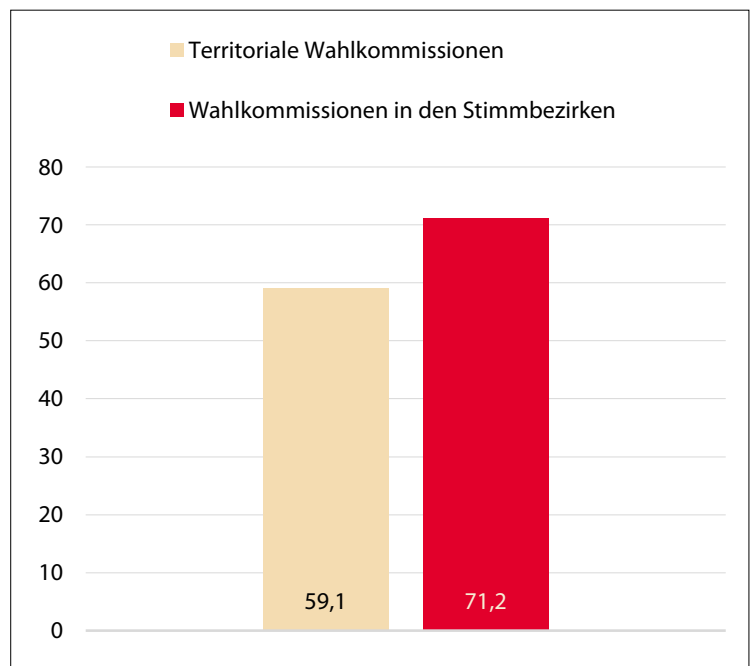

Quelle: OSCE/ODIHR Election Observation Mission Final Report, Presidential Elections, 11 October 2015, Republic of Belarus, S. 9, <https://www.osce.org/odihr/ elections/287486?download=true> 
Grafik 8: Anteil der Frauen in den Kommunalräten (Stand: März 2018)

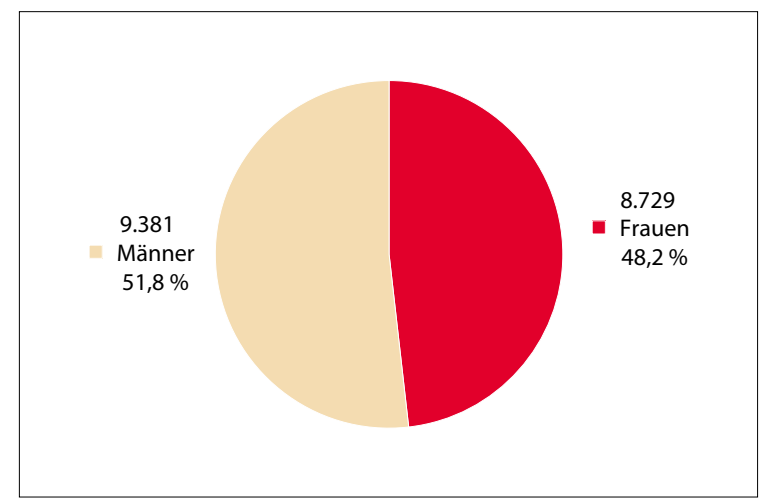

Quelle:Zentrale Wahlkommission der Republik Belarus: Swedenija o sostawe isbrannych deputatow mestnych Sowetow deputatow dwadzat wosmogo sosywa, <http://rec.gov.by/sites/default/ files/pdf/Elections-MS28-elect_59-60.pdf>

Grafik 10: Anteil der Frauen in leitenden Positionen der Ministerien (Stand: März 2018)

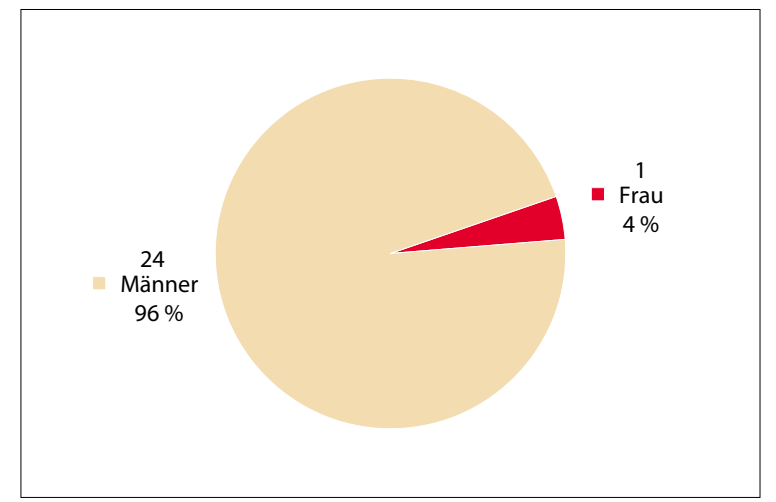

Quelle: Minsterrat der Republik Belarus: Ministerstwa, <http:// www.government.by/ru/departments/>
Grafik 9: Anteil der Frauen im Repräsentantenhaus der Nationalversammlung der Republik Belarus (Stand: März 2018)

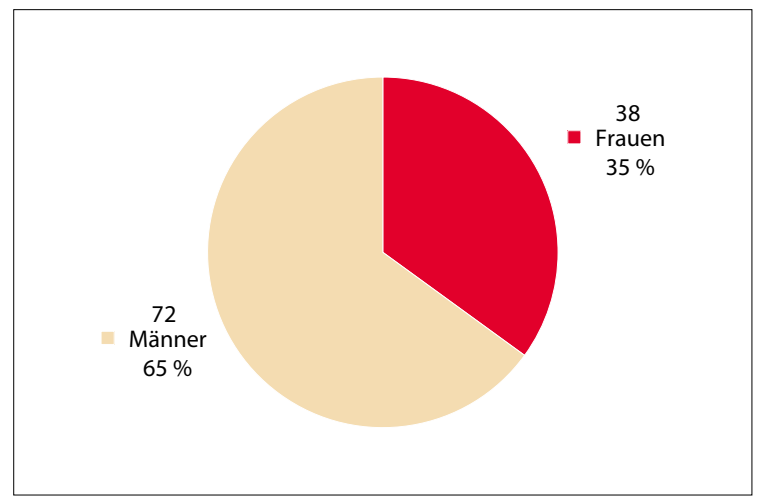

Quelle: Parlament der Republik Belarus: Deputatskij korpus, $<$ http://www.house.gov.by/ru/deputies-ru/> 


\section{Bericht unabhängiger Wahlbeobachter zu den Kommunalwahlen in Belarus}

\section{Local elections in Belarus: Final report (by Human Rights Defenders for Free Elections*)}

*The campaign of election observation during the 2018 elections to local councils was carried out by the Human Right Center "Viasna" and the Belarusian Helsinki Committee. The campaign was aimed at evaluating the elections from the viewpoint of the Belarusian electoral legislation and international standards of free and democratic elections, as well as informing the Belarusian public and international community about the progress of the elections and results of observation.

\section{Findings and conclusions}

Elections of deputies of local councils of the $28^{\text {th }}$ convocation, which began on November 14, 2017, took place against the background of an overall deterioration of the political situation in the country compared to the previous period of 'soft practices' lasting between August 2015 and February 2017. The period ended with a wave of protests caused by the application of Decree number 3, which was perceived by the authorities as a serious threat to political stability and resulted in repression against peaceful protesters, opposition activists and leaders, civil society members, independent journalists and human rights defenders. Politically motivated persecution was used, in one form or another, against over 900 people. Forty people were victims of politically motivated criminal prosecution.

Despite the fact that the overall situation stabilized by the year-end, the country's prisons continued to hold political prisoners Mikhail Zhamchuzhny and Dzmitry Paliyenka. The latter is a prisoner of conscience, according to Amnesty International. The authorities failed to drop criminal charges against activists of the independent trade union REP, Henadz Fiadynich and Ihar Komlik. In December 2017 and January 2018, the Ministry of Information blocked access to two popular independent online resources, Belarusian Partisan (www.belaruspartisan.org) and Charter 97 (www. charter97.org). Judicial and other harassment was still used against independent journalists working with foreign media without government accreditation (most notably, TV channel Belsat).

After the launch of the elections, the Belarusian authorities said that they were not going to finalize the process of further improvement of the electoral legislation in line with the recommendations of the OSCE ODIHR. Thus, the electoral law was not reformed and the elections to local councils were governed by the old rules and procedures, which have been repeatedly criticized, including by the OSCE ODIHR observers.

The elections were very passive and hardly visible to the public. Despite the absence of any significant violations and obstacles at the electoral phases of registration of nomination groups, collecting of signatures and election campaigning, the stages that followed, including early voting, counting of the votes and the tabulation of voting results in higher commissions, were marred by numerous manipulations, active use of administrative resources and lack of transparency.

Monitoring of all stages of the elections once again clearly emphasized the systemic problems inherent in the electoral process that need to be addressed.

The electoral process did not comply with a number of key international standards for democratic and free elections. This is evidenced by the lack of equal access to the media for all candidates, the absence of impartial electoral commissions, numerous cases of voter coercion to participate in early voting, and opacity of some election procedures for observers. The key reason for criticism is the lack of transparency of the vote count, which prevents the observers from viewing the announced election results as a reflection of the will of voters.

\section{Election commissions}

The formation of election commissions at all levels took place in an atmosphere of greater openness, as compared to the last local elections in 2014. All the representatives of the campaign "Human Rights Defenders for Free Elections" had the opportunity to attend meetings of the authorities in charge of the formation of election commissions, but in most cases the campaign's observers were denied access to the nomination documents, which were submitted to local executive and regulatory bodies.

The formation of election commissions, as in previous elections, was marked by a discriminatory approach towards representatives of the opposition parties in comparison with the nominees from the pro-government political parties and public associations, as well as labor collectives. In particular, only $20.6 \%$ of the nominees from the opposition 
parties were included in the territorial election commissions (TECs) $(0.067 \%$ of the total number of TEC members), $16.7 \%$ — in the district election commissions (DECs) ( $0.53 \%$ of the total number of DEC members), and $11 \%$ - in the precinct election commissions (PECs) $(0.04 \%$ the total number of PEC members). At the same time, over $90 \%$ of representatives of the pro-government political parties and the five biggest pro-government public associations (Belaya Rus, Belarusian Republican Youth Union, Belarusian Women's Union, Belarusian Public Association of Veterans, and the Federation of Trade Unions of Belarus) won seats on the commissions.

Absence of specific criteria for the selection of candidates negate all efforts to appeal against the decisions of the bodies in charge of forming the commissions.

Representation of political parties in election commissions is still extremely low, reflecting the specifics of the Belarusian political system. The main actors of the election process (including election officials) are pro-governmental associations and labor groups.

\section{Nomination and registration of candidates}

According to the CEC, 17,542 nomination groups were registered, which is $99.9 \%$ of the total number of nominees.

Statistics of refusals to register and withdrawn nominations demonstrate the level of applying the technical procedures of registration (verification of signatures, declarations, etc.) in the political struggle. The audits were used selectively, primarily against opposition candidates. The number of refusals to register candidates for the Minsk City Council was $23 \%$, the Councils at the district level $-1.5 \%$, the rural councils $-0.3 \%$.

$78 \%$ of the campaign's observers were not admitted directly to the verification procedures and were not able to report a comprehensive and unbiased approach by the commissions. The observers were only able to attend the meetings where the results of verification were announced and decisions on registration were taken. This significantly reduces credibility of the work of election officials, calls into question not only individual decisions to refuse the registration of candidates, but also the decisions which allowed their registration.

\section{Election campaigning}

In comparison with the previous elections of local councils of deputies, this year's elections were marked by a greater number of locations for campaigning events, meetings with voters and posting campaign materials. These venues were also more convenient. When making decisions about campaigning locations, local governments were increasingly guided by the principle "everything which is not forbidden is allowed."

Much more candidates, as compared to the previous elections, filed notices of events under a simplified procedure. Accordingly, the number of announced election pickets increased. Traditionally, election campaigning events were more active in Minsk: one candidate announced an average of about 70 events.

Election commissions in the regions mainly failed to inform the public about the time and place of the meetings with voters. 30\% of the campaign's observers in the regions reported that candidates were deprived of equal opportunities and could not meet with voters in the premises provided by the authorities. As during previous election campaigns, administrative resources were extensively used in favor of pro-government candidates.

There were no significant barriers to electoral pickets; however, there were elements of pressure at the pickets of opposition figures.

\section{Early voting}

As before, early voting was orchestrated by local authorities, administrations of government-owned enterprises and institutions. In some cases, the use of administrative resources in order to ensure voter turnout was accompanied by elements of control of the voters (forcing them to come or not to come to the polls) and threats of various disciplinary sanctions for those who do not want to take part in the elections.

Early voting remains one of the key areas of concern in the overall electoral process in Belarus.

\section{Home voting}

Home voting was accompanied by significant violations of the procedure under Art. 54 of the Electoral Code. The campaign's observers documented cases of voters' complaints to the PECs alleging that they did not request home voting.

$33.3 \%$ of the observers reported cases when voters said, when visited by the PEC members, that they had not applied for arranging home voting.

$50 \%$ of the observers noted the discrepancy between the number of home voters and the number of ballots issued to the PEC members to organize such a vote. 


\section{Voting at the polling stations and vote count}

The Electoral Code does not describe the procedure of ballot counting. Recommendations of the OSCE ODIHR and the proposals of the campaign "Human Rights Defenders for Free Elections" on the exact and detailed resolution of the procedure through a decision of the CEC were not been taken into account during the election preparations.

The campaign's observers point out that the vast majority of the PECs covered by the observation carried out a joint and simultaneous counting of ballots, without announcing the voter's choice and demonstrating each ballot to those present. Such a procedure of counting of the ballots is not transparent and does not allow to correlate the results of observation to the data reflected in the protocol of voting results. $97 \%$ of the observers assessed the vote count as opaque.

During the observation of the counting procedures, the campaign's observers documented other violations of electoral laws: $7.1 \%$ of the PECs did not carry out a separate counting of votes, $42.3 \%$ of the PECs did not announce the results of the separate counting of votes, $53.6 \%$ of the observers were seated at a considerable distance from the tables on which the vote count was carried out, which prevented them from conducting a comprehensive observation of the procedure.

\section{Appeals against violations of electoral law}

Filing appeals against decisions and actions of commissions and other participants in the electoral process still fails to bring positive results, which makes actors in the electoral process rarely use the tool in practice.

Source: Local elections. Final report, <https://elections2018.spring96.org/en/news/89248> 


\section{Dezember 2017 - 5. März 2018}

\begin{tabular}{|c|c|}
\hline 06.12 .2017 & $\begin{array}{l}\text { Der Außenminister von Belarus, Uladsimir Makej, nimmt am 24. OSZE-Außenministertreffen } \\
\text { in Wien teil und spricht u. a. über die Krise der europäischen Sicherheit. Das Potential der OSZE } \\
\text { müsse dazu genutzt werden, die Risiken der militärischen Konfrontation in Europa zu senken, } \\
\text { erklärt Makej. Belarus sei bereit, bei der Schaffung einer neuen zeitgemäßen Kontrolle über kon- } \\
\text { ventionelle Waffen mitzuwirken, so der Außenminister. }\end{array}$ \\
\hline 08.12 .2017 & $\begin{array}{l}\text { Der Außenminister von Belarus, Uladsimir Makej, sagt in einem Interview gegenüber dem rus- } \\
\text { sischen TV-Sender »Nastojaschtscheje wremja«, dass die Länder des Westens ein unabhängiges } \\
\text { und souveränes Belarus brauchen. Belarus wiederum sei an den guten Beziehungen mit der EU, } \\
\text { der EAWU und mit Russland interessiert. Geographisch wie politisch gehöre Belarus zu Europa, } \\
\text { so Makej. }\end{array}$ \\
\hline 10.12 .2017 & $\begin{array}{l}\text { Der Vorsitzende der Menschenrechtsorganisation »Belarussisches Helsinki-Komitee«, Aleh Hulak, } \\
\text { erklärt aus Anlass des Internationalen Tags der Menschenrechte, der am 10. Dezember began- } \\
\text { gen wird, es habe im Jahr } 2017 \text { keine erheblichen Veränderungen in der Menschenrechtssitua- } \\
\text { tion in Belarus gegeben. }\end{array}$ \\
\hline 11.12 .2017 & $\begin{array}{l}\text { Der stellvertretende Außenminister von Belarus, Aleh Krautschanka, erklärt während des Forums } \\
\text { „Europäische Union und Östliche Partnerschaft: Ansätze für regionale Zusammenarbeit« in } \\
\text { Minsk, Belarus plane nicht, Mitglied der Europäischen Union zu werden, sei allerdings an der } \\
\text { Teilnahme an der Östlichen Partnerschaft der EU interessiert. }\end{array}$ \\
\hline 18.12 .2017 & $\begin{array}{l}\text { Der Außenminister von Litauen, Linas Linkevičius, erklärt während eines Treffens einer ressort- } \\
\text { übergreifenden Arbeitsgruppe für Strahlenschutz und nuklearen Sicherheit, dass das Projekt des } \\
\text { belarussischen Atomkraftwerks in Astrawez weniger ökonomisch, sondern nur politisch motiviert } \\
\text { sei. Belarus brauche nicht derart viel Energie, und das AKW gefährde die Umwelt und nukleare } \\
\text { Sicherheit in der Region, so Linkevičius. }\end{array}$ \\
\hline 19.12 .2017 & $\begin{array}{l}\text { Belarus stimmt bei der Verabschiedung einer Resolution zur »Lage der Menschenrechte in der } \\
\text { Autonomen Republik Krim und der Stadt Sewastopol (Ukraine)" durch die UN-Generalver- } \\
\text { sammlung mit Nein. Der belarussische Außenminister Uladsimir Makej erklärt, dass Belarus } \\
\text { generell gegen alle landesspezifische Resolutionen eintrete, weil sie nicht konstruktiv seien. }\end{array}$ \\
\hline 19.-20.2017 & $\begin{array}{l}\text { Der Generaldirektor des Europarats für Menschenrechte und Rechtsstaatlichkeit, Christos Gia- } \\
\text { koumopoulos, hält sich zu einem Arbeitsbesuch in Belarus auf. Giakoumopoulos stellt fest, dass } \\
\text { das Niveau der Zusammenarbeit zwischen dem Europarat und Belarus in den letzten Jahren erheb- } \\
\text { lich zugenommen habe. Er äußert die Ansicht, die belarussische Gesellschaft sei für die Abschaf- } \\
\text { fung der Todesstrafe bereit. }\end{array}$ \\
\hline 19.-20.12.2017 & $\begin{array}{l}\text { In Brüssel findet die 4. Sitzung der } 2016 \text { gegründeten Koordinierungsgruppe Belarus-EU statt. } \\
\text { Die belarussische Delegation wird vom stellvertretenden Außenminister, Aleh Krautschanka, } \\
\text { geleitet. Im Mittelpunkt der Gespräche stehen folgende Themen: der Dialog Belarus-EU, die Mit- } \\
\text { gliedschaft von Belarus in der Östlichen Partnerschaft, die Kooperation im Bereich der Mobili- } \\
\text { tät, der aktuelle Handelsdialog, die Zusammenarbeit im Rahmen der technischen Hilfe der EU } \\
\text { und die Wahrung der Menschenrechte. }\end{array}$ \\
\hline 20.12 .2017 & $\begin{array}{l}\text { Die trilaterale Kontaktgruppe zur Beilegung der Krise im Osten der Ukraine tagt in Minsk. Der } \\
\text { Sonderbeauftragter des OSZE-Vorsitzenden in der Gruppe, Martin Sajdik, bedankt sich beim } \\
\text { belarussischen Außenministerium für die »hervorragende Arbeitsbedingungen« im Jahr } 2017 .\end{array}$ \\
\hline 21.12 .2017 & $\begin{array}{l}\text { Der belarussische Präsident Aljaksandr Lukaschenka unterzeichnet das Dekret "Über die Ent- } \\
\text { wicklung der digitalen Wirtschaft«. Damit werde Belarus zum ersten Land in der Welt, das eine } \\
\text { breite Verwendung von Blockchain-Technologien ermöglicht, so Lukaschenka. }\end{array}$ \\
\hline 22.12 .2017 & $\begin{array}{l}\text { Die EU verurteilt die zwei Todesurteile, die am 20.12. } 2017 \text { gegen Sjamjon Beraschnoj und Ihar } \\
\text { Herschankou verhängt wurden, und ruft Belarus erneut zur Einführung eines Moratoriums für } \\
\text { die Todesstrafe auf. }\end{array}$ \\
\hline
\end{tabular}




\begin{tabular}{|c|c|}
\hline 26.12.2017 & $\begin{array}{l}\text { Die Dauer des visafreien Aufenthalts von Ausländern in bestimmten Gebieten der Regionen } \\
\text { Hrodna und Brest wird ab dem 1. Januar } 2018 \text { von } 5 \text { auf } 10 \text { Tage erhöht. }\end{array}$ \\
\hline 01.01 .2018 & $\begin{array}{l}\text { Der belarussische Außenminister Uladsimir Makej erklärt in einem Interview gegenüber dem } \\
\text { belarussischen TV-Sender "Belarus-1«, die Wahrnehmung von Belarus durch westliche Länder } \\
\text { und Ansätze zur Entwicklung der Zusammenarbeit von Belarus mit diesen Ländern hätten sich } \\
\text { positiv verändert. }\end{array}$ \\
\hline 04.01 .2018 & $\begin{array}{l}\text { In einem Bericht des belarussischen Außenministeriums zur Außenpolitik des Landes im Jahr } 2017 \\
\text { werden u. a. folgende Ergebnisse aufgeführt: Russland sei Hauptpartner im Handel und Investi- } \\
\text { tionen geblieben; die Beziehungen mit den USA hätten sich fortschreitend weiterentwickelt; der } \\
\text { Dialog mit der EU habe mehr Struktur und wirtschaftliche Substanz erhalten. }\end{array}$ \\
\hline 11.01 .2018 & $\begin{array}{l}\text { Die Verhandlungen zwischen Belarus und der EU über die Visa- und Rückübernahmeabkommen } \\
\text { befinden sich in der Endphase, teilt der belarussische Uladsimit Makej mit. Gleichzeitig werden } \\
\text { mit der Russischen Föderation Verhandlungen zum Abkommen über die gegenseitige Anerken- } \\
\text { nung von Visa durchgeführt, so Makej. }\end{array}$ \\
\hline 15.01 .2018 & $\begin{array}{l}\text { Vertreter belarussischer Menschenrechtsorganisationen richten eine gemeinsame Erklärung an } \\
\text { westliche Diplomaten mit der Bitte, die belarussischen Behörden dazu aufzufordern, die grau- } \\
\text { same Behandlung von Michail Shamtschushny zu stoppen. Shamtschushny ist ehemaliger Dozent } \\
\text { einer Universität in Wizebsk und wurde } 2015 \text { wegen "vorsätzlicher Offenlegung von Informa- } \\
\text { tionen, die ein Dienstgeheimnis darstellten, sowie wegen Erwerbs oder Herstellung von Mitteln } \\
\text { zur heimlichen Erlangung von Informationen« verurteilt. Den Angaben der Menschenrechtlern } \\
\text { zufolge hatte er Informationen über Haftbedingungen in Belarus weitergegeben. Belarussische } \\
\text { Menschenrechtsorganisationen bezeichnen Shamtschushny als politischen Gefangenen. }\end{array}$ \\
\hline 16.01 .2018 & $\begin{array}{l}\text { In der neu veröffentlichen Liste über die Lage der bürgerlichen und politischen Freiheiten von } \\
\text { «Freedom House» wird Belarus nach wie vor in der Gruppe der nicht freien Länder platziert. }\end{array}$ \\
\hline 18.01 .2018 & Die trilaterale Kontaktgruppe zur Beilegung der Krise im Osten der Ukraine tagt erneut in Minsk. \\
\hline 19.01.2018 & $\begin{array}{l}\text { Zwei freiberufliche Journalisten und ein Kameramann - Kastus Shukouski, Volha Tschajtschyz } \\
\text { und Andrej Toltschyn - werden für ihre Kooperation mit dem in Belarus nicht akkreditierten } \\
\text { polnischen TV-Sender »Belsat« zu Geldstrafen verurteilt. Nach Angaben des Belarussischen Jour- } \\
\text { nalistenverbands BAJ, hat es im Jahr } 2017 \text { insgesamt } 69 \text { derartige Verurteilungen von belarussi- } \\
\text { schen freien Journalisten wegen ihrer Kooperation mit ausländischen, in Belarus nicht akkredi- } \\
\text { tierten Medien gegeben. }\end{array}$ \\
\hline 20.01.2018 & $\begin{array}{l}\text { In Belarus wird gegen zwei u. a. wegen Mordes Angeklagte - Aljaksandr Shilnikau und Wjat- } \\
\text { scheslau Sucharka - von einem Minsker Gericht die Todestrafe verhängt. }\end{array}$ \\
\hline 23.01.2018 & $\begin{array}{l}\text { Die Europäische Union ruft in einer Erklärung Belarus dazu auf, ein Moratorium auf die Vollstre- } \\
\text { ckung der Todesstrafe zu verkünden und alle ergangenen Todesurteile in mildere Strafen umzu- } \\
\text { wandeln. Auch die Parlamentarische Versammlung des Europarates (PACE) verurteilt am fol- } \\
\text { genden Tag die Urteile. }\end{array}$ \\
\hline 22.-24.01.2018 & $\begin{array}{l}\text { Der Sonderbeauftragte der Parlamentarischen Versammlung der OSZE für Osteuropa und Lei- } \\
\text { ter der Delegation des schwedischen Parlaments bei der Parlamentarischen Versammlung der } \\
\text { OSZE, Kent Harsted, hält sich zu einem Arbeitsbesuch in Belarus auf. Er trifft sich u. a. mit } \\
\text { Präsident Aljaksandr Lukaschenka, Außenminister Uladsimir Makej, der Leiterin der Zentra- } \\
\text { len Wahlkommission, Lidsija Jarmoschyna, Vertretern des Parlaments sowie der Opposition und } \\
\text { der Zivilgesellschaft. Harsted äußert sein Bedauern darüber, dass der Reformprozess der Wahl- } \\
\text { gesetzgebung in Belarus nicht voranschreite, und fordert die belarussischen Behörden zu inten- } \\
\text { siveren Reformen auf. }\end{array}$ \\
\hline $24 .-25.01 .2018$ & $\begin{array}{l}\text { Eine Delegation der »Europäischen Volkspartei« hält sich im Rahmen des Monitorings der Kom- } \\
\text { munalwahlen in Belarus zu einem Besuch auf. Die Wahlen sind für den 13.-18. Februar ange- } \\
\text { setzt. Die Delegationsmitglieder treffen sich u. a. mit der Leiterin der EU-Delegation in Belarus, } \\
\text { Andrea Wiktorin, sowie mit Vertretern der Opposition. }\end{array}$ \\
\hline
\end{tabular}




\begin{tabular}{|c|c|}
\hline 30.01 .2018 & $\begin{array}{l}\text { Der EU-Kommissar für Europäische Nachbarschaftspolitik und Erweiterungsverhandlungen, } \\
\text { Johannes Hahn, hält sich zu einem Arbeitsbesuch in Belarus auf. Dabei werden Gespräche u. a. } \\
\text { mit Präsident Aljaksandr Lukaschenka, Außenminister Uladsimir Makej sowie mit Vertretern } \\
\text { der Opposition geführt. Gesprächsthemen sind u. a. die bilateralen Beziehungen zwischen der } \\
\text { EU und Belarus, effektive Staatsführung und -entwicklung, Verkehr, Energieeffizienz und die } \\
\text { Bekämpfung des Klimawandels, zwischenmenschliche Kontakte, Menschenrechtsfragen sowie } \\
\text { der Wahlprozess in Belarus. Die EU strebe eine Stärkung der Beziehungen zu Belarus an, erklärt } \\
\text { Hahn. Belarus sei an einer starken EU interessiert, so Lukaschenka. }\end{array}$ \\
\hline 31.01.2018 & $\begin{array}{l}\text { Die trilaterale Kontaktgruppe zur Beilegung der Krise im Osten der Ukraine tagt ein weiteres } \\
\text { Mal in Minsk. }\end{array}$ \\
\hline 02.02 .2018 & $\begin{array}{l}\text { Drei belarussische Autoren der russischen Nachrichtenagentur »Regnum»- Juryj Paulawez, Sjar- } \\
\text { hej Schyptenka und Dzmitryj Alimkin - werden von einem Minsker Gericht zu fünf Jahren } \\
\text { Haft mit einer Aussetzung des Strafvollzugs für drei Jahre verurteilt. Alle drei Autoren wurden } \\
\text { im Dezember } 2016 \text { in Minsk verhaftet und beschuldigt, in ihren prorussischen Artikeln gemein- } \\
\text { schaftlich ethnischen Hass angefacht zu haben (Volksverhetzung). }\end{array}$ \\
\hline 08.02 .2018 & $\begin{array}{l}\text { Präsident Aljaksandr Lukaschenka trifft sich in Minsk mit dem Ministerpräsidenten Lettlands, } \\
\text { Māris Kučinskis, um die allgemeinen bilateralen Beziehungen zu erörtern. }\end{array}$ \\
\hline 02.02 .2018 & $\begin{array}{l}\text { Die EU verurteilt in einer Erklärung erneut ein Todesurteil, das gegen Wiktar Ljotau im Septem- } \\
\text { ber } 2017 \text { verhängt wurde. Die Verurteilung war erst im Januar } 2018 \text { bekannt geworden. Es wird } \\
\text { erneut zur Einführung eines Moratoriums auf die Todesstrafe aufgerufen. }\end{array}$ \\
\hline 08.02.2018 & $\begin{array}{l}\text { Belarus wolle zum regionalen Spitzenreiter im IT-Bereich werden, erklärt der belarussische Außen- } \\
\text { minister Uladsimir Makei im Interview gegenüber der russischen Zeitung »Iswestija». }\end{array}$ \\
\hline 13.-18.02.2018 & In Belarus finden Kommunalwahlen statt. \\
\hline 15.02 .2018 & $\begin{array}{l}\text { Präsident Aljaksandr Lukaschenka trifft sich in Minsk mit dem Ministerpräsidenten der Türkei, } \\
\text { Binali Yıldırım. Die belarussisch-türkischen politischen und wirtschaftlichen Beziehungen ste- } \\
\text { hen im Mittelpunkt des Gesprächs. }\end{array}$ \\
\hline 16.-18.02.2018 & $\begin{array}{l}\text { Der belarussische Außenminister Uladsimir Makej nimmt an der 54. Münchner Sicherheitskon- } \\
\text { ferenz teil. Unter den Themen der Konferenz sind die „Helsinki-2 Initiative«, die Entsendung von } \\
\text { Friedenstruppen in die Ostukraine und die Europäischen Spiele-2019 in Belarus. Am Rande der } \\
\text { Konferenz trifft sich Makej mit dem Vorsitzenden der Konferenz, Wolfgang Ischinger, und mit } \\
\text { dem Sonderbeauftragten des US-Außenministeriums für Verhandlungen zur Ukraine, Kurt Volker. }\end{array}$ \\
\hline 19.02 .2018 & $\begin{array}{l}\text { Außenminister Makej erklärt, Belarus sei bereit, Friedenstruppen in den Donbass zu entsenden, } \\
\text { sollte dies notwendig werden. }\end{array}$ \\
\hline 19.02.2018 & $\begin{array}{l}\text { Nach Angaben der Kampagne »Menschenrechtler für faire Wahlen« entsprechen die offiziellen } \\
\text { Wahlergebnisse bei den Kommunalwahlen nicht den tatsächlichen. } 789 \text { Wahlbeobachter der } \\
\text { Kampagne hatten landesweit an der Beobachtung teilgenommen. }\end{array}$ \\
\hline 20.02 .2018 & $\begin{array}{l}\text { Die Ko-Vorsitzende der Bewegung »Für die Freiheit« Tazjana Karatkewitsch äußert sich zur Oppo- } \\
\text { sition in Belarus: Diese befinde sich in einer Krise, und die mangelnde aktive Beteiligung an den } \\
\text { Kommunalwahlen sei eine Bestätigung dafür. }\end{array}$ \\
\hline 20.02 .2018 & $\begin{array}{l}\text { In einer Erklärung des Pressesprechers des Europäischen Auswärtigen Dienstes im Zusammen- } \\
\text { hang mit den Kommunalwahlen in Belarus wird festgestellt, dass eine Reihe der Schlüsselemp- } \\
\text { fehlungen von OSZE/ODIHR und des Europarates bei den Wahlen nicht berücksichtigt wurden. }\end{array}$ \\
\hline 21.02 .2018 & $\begin{array}{l}\text { Ein Erreichen der Ziele der nachhaltigen Entwicklung könne zu einem Fortschritt im Bereich } \\
\text { der Menschenrechte in Belarus beitragen, erklärt die Leiterin der EU-Delegation in Belarus, } \\
\text { Andrea Wiktorin. }\end{array}$ \\
\hline 21.02 .2018 & $\begin{array}{l}\text { Präsident Aljaksandr Lukaschenka trifft sich mit der Ersten Stellvertretenden Generalsekretärin } \\
\text { der Vereinten Nationen, Amina Mohammed, in Minsk. }\end{array}$ \\
\hline
\end{tabular}




\begin{tabular}{|l|l|}
\hline 21.-22.02.2018 & $\begin{array}{l}\text { In Minsk findet das Regionalforum der nationalen Koordinatoren zur Erreichung der Nachhal- } \\
\text { tigkeitsziele (SDG) statt, das vom belarussischen Außenministerium und der Organisation der } \\
\text { Vereinigten Nationen veranstaltet wird. Das Forum versammelt Delegationen aus über 40 Staa- } \\
\text { ten, darunter hochrangige Beamten und Vertreter internationaler Organisationen. Die Teilneh- } \\
\text { mer des Forums tauschen ihre Erfahrungen bei der Umsetzung der SDG aus, besprechen Pro- } \\
\text { bleme bei der Erfüllung der Agenda für nachhaltige Entwicklung bis 2030. }\end{array}$ \\
\hline 21.-24.02.2018 & $\begin{array}{l}\text { Eine parlamentarische Delegation von Belarus mit dem Vizepräsidenten des Repräsentanten- } \\
\text { hauses der Nationalversammlung, Baleslau Pirschtuk, an der Spitze nimmt an der Wintertagung } \\
\text { der Parlamentarischen Versammlung der OSZE in Wien teil. Es wird u. a. über die Entwick- } \\
\text { lung langfristiger Strategien zur Bekämpfung des Klimawandels, über die Rüstungskontrolle im } \\
\text { OSZE-Raum sowie die Förderung der Demokratie diskutiert. }\end{array}$ \\
\hline 23.02.2018 & $\begin{array}{l}\text { Der Rat der EU verlängert die verbliebenen Sanktionen gegen Belarus um ein weiteres Jahr (bis } \\
\text { zum 28. Februar 2019). Es handelt sich um ein Waffenembargo sowie das Einfrieren der Vermö- } \\
\text { genswerte und ein Reiseverbot für vier Personen, die mit dem ungeklärten Verschwinden zweier } \\
\text { Oppositionspolitiker, eines Geschäftsmanns und eines Journalisten, in den Jahren 1999 und 2000 } \\
\text { in Zusammenhang gebracht werden. }\end{array}$ \\
\hline 28.02.2018 & $\begin{array}{l}\text { Die trilaterale Kontaktgruppe zur Beilegung der Krise im Osten der Ukraine tagt erneut in Minsk. } \\
\text { Konferenz zur Östlichen Partnerschaft der EU in Tallinn teil, die durch das Estnische Zentrum } \\
\text { der Östlichen Partnerschaft gemeinsam mit der Europäischen Kommission ausgerichtet wird. }\end{array}$ \\
\hline 02.03.2018
\end{tabular}

Zusammengestellt auf der Grundlage der Meldungen der Nachrichten-Agenturen BelaPAN und BelTa und der Homepage naviny. by.

Sie können die gesamte Chronik ab dem 14.03.2011 auch auf<http://www.laender-analysen.de/belarus/> unter dem Link »Chronik«lesen.

Herausgeber:

Forschungsstelle Osteuropa an der Universität Bremen

Deutsche Gesellschaft für Osteuropakunde e.V.

Deutsches Polen-Institut

Leibniz-Institut für Agrarentwicklung in Transformationsökonomien

Leibniz-Institut für Ost- und Südosteuropaforschung

Zentrum für Osteuropa- und internationale Studien (ZOiS) g GmbH

Redaktion:

Olga Dryndova (verantwortlich)

Sprachredaktion: Hartmut Schröder

Satz: Matthias Neumann

Wissenschaftlicher Beirat:

Prof. Dr. Margarita Balmaceda, Seton Hall University / Harvard University

Dr. Maria Davydchyk, Deutsche Gesellschaft für Auswärtige Politik, Berlin

Prof. Dr. Heiko Pleines, Forschungsstelle Osteuropa an der Universität Bremen Dr. Astrid Sahm, IBB Dortmund

Die Meinungen, die in den Belarus-Analysen geäußert werden, geben ausschließlich die Auffassung der Autoren wieder

Abdruck und sonstige publizistische Nutzung sind nach Rücksprache mit der Redaktion gestattet. Belarus-Analysen-Layout: Cengiz Kibaroglu, Matthias Neumann und Michael Clemens

Alle Ausgaben der Belarus-Analysen sind mit Themen- und Autorenindex archiviert unter www.laender-analysen.de ISSN 2192-1350 @ 2018 by Forschungsstelle Osteuropa an der Universität Bremen 

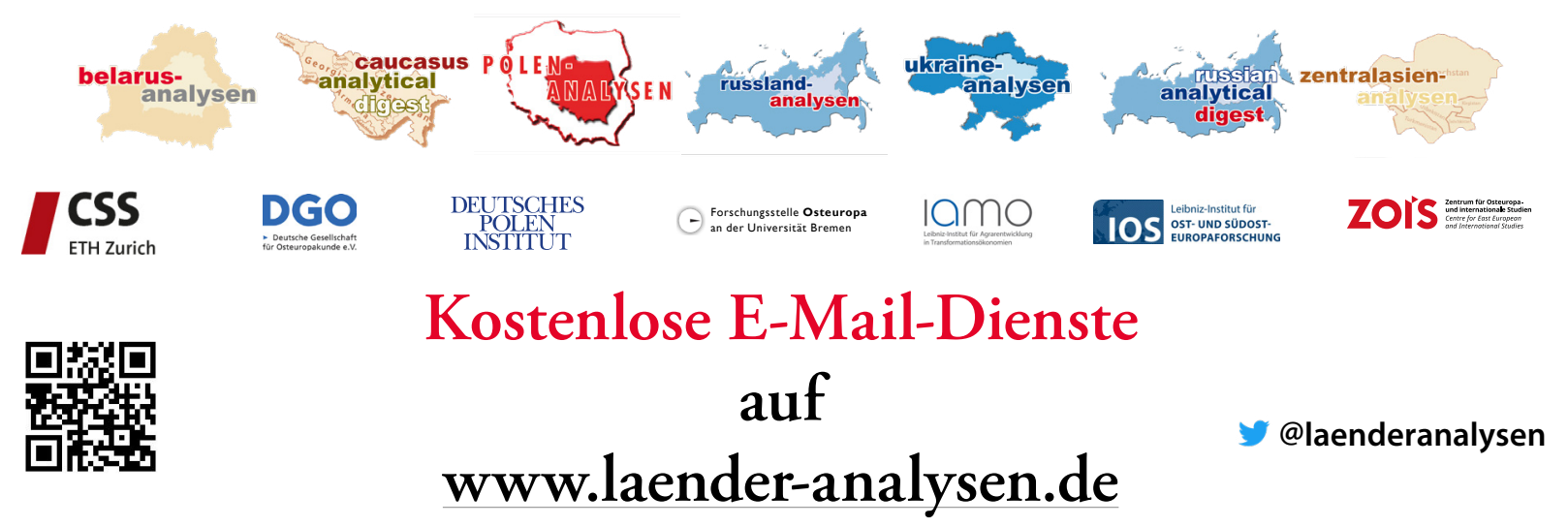

Die Länder-Analysen bieten regelmäßig im kostenlosen Abonnement kompetente Einschätzungen aktueller politischer, wirtschaftlicher, sozialer und kultureller Entwicklungen in Ostmitteleuropa und der GUS. Alle Länder-Analysen verstehen sich als Teil eines gemeinsamen Projektes, das der wissenschaftlich fundierten, allgemeinverständlich formulierten Analyse der Entwicklungen im östlichen Europa, der Offenheit für verschiedene inhaltliche Positionen und der kostenlosen und nicht-kommerziellen Information einer breit verstandenen interessierten Öffentlichkeit verpflichtet ist. Autor/innen sind internationale Fachwissenschaftler/innen und Expert/innen. Die Redaktionen der Länder-Analysen bestehen aus Wissenschaftler/innen mit langjähriger Forschungserfahrung.

Die deutschsprachigen Länder-Analysen werden gemeinsam von der Forschungsstelle Osteuropa an der Universität Bremen, dem Zentrum für Osteuropa- und internationale Studien, der Deutschen Gesellschaft für Osteuropakunde, dem Deutschen Polen-Institut, dem Leibniz-Institut für Agrarentwicklung in Transformationsökonomien und dem Leibniz-Institut für Ost- und Südosteuropaforschung herausgegeben. Die englischsprachigen Länder-Analysen erscheinen in Kooperation der Forschungsstelle Osteuropa mit dem Center for Security Studies (CSS) der ETH Zürich.

Die Länder-Analysen bieten regelmäßig Kurzanalysen zu aktuellen Themen, ergänzt um Grafiken und Tabellen sowie Dokumentationen. Zusätzlich gibt es eine Chronik aktueller Ereignisse. Alle Länder-Analysen sind auch mit Archiv und Indizes online verfügbar unter $<$ www.laender-analysen.de $>$.

\section{Belarus-Analysen \\ Erscheinungsweise: zweimonatlich \\ Abonnement unter: <http://www.laender-analysen.de/belarus/>}

\section{Caucasus Analytical Digest}

In englischer Sprache. Erscheinungsweise: monatlich

Abonnement unter: <http://www.css.ethz.ch/en/publications/cad.html>

\section{Polen-Analysen}

Erscheinungsweise: zweimal monatlich

Abonnement unter: <http://www.deutsches-polen-institut.de/newsletter/polen-analysen/>

Auch als App für Android ${ }^{\mathrm{TM}}$ (ab Januar 2016) kostenlos auf Google Play ${ }^{\mathrm{TM}}$.

\section{Russland-Analysen}

Erscheinungsweise: zweiwöchentlich

Abonnement unter: <http://www.laender-analysen.de/russland/>

Auch als App für Android (ab Januar 2016) kostenlos auf Google Play.

\section{$D$ Google Play}

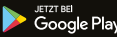

\section{Russian Analytical Digest}

In englischer Sprache. Erscheinungsweise: zweimal monatlich

Abonnement unter: $<$ http://www.css.ethz.ch/en/publications/rad.html>

\section{Ukraine-Analysen}

Erscheinungsweise: zweimal monatlich

Abonnement unter: <http://www.laender-analysen.de/ukraine/>

Auch als App für Android (ab Januar 2016) kostenlos auf Google Play.

\section{Zentralasien-Analysen}

Erscheinungsweise: monatlich

Abonnement unter: <http://www.laender-analysen.de/zentralasien/>

Auch als App für Android (ab Januar 2016) kostenlos auf Google Play. 